\title{
NEARBY SUPERNOVA FACTORY OBSERVATIONS OF SN 2007if: FIRST TOTAL MASS MEASUREMENT OF
} A SUPER-CHANDRASEKHAR-MASS PROGENITOR

\author{
R. A. Scalzo ${ }^{1,14}$, G. Aldering ${ }^{2}$, P. Antilogus ${ }^{3}$, C. Aragon ${ }^{2}$, S. Bailey ${ }^{3}$, C. Baltay ${ }^{1}$, S. Bongard ${ }^{3}$, C. Buton B $^{4,5,6}$,

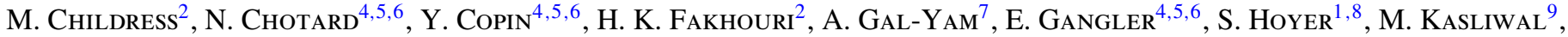 \\ S. Loken ${ }^{2}$, P. Nugent ${ }^{10}$, R. Pain ${ }^{3}$, E. Pécontal ${ }^{11}$, R. Pereira ${ }^{4,5,6}$, S. Perlmutter ${ }^{2}$, D. Rabinowitz ${ }^{1}$, A. Rau ${ }^{9}$, \\ G. Rigaudier $^{11}$, K. Runge ${ }^{2}$, G. Smadja ${ }^{4}$, C. TaO ${ }^{12}$, R. C. Thomas ${ }^{10}$, B. Weaver ${ }^{13}$, and C. Wu \\ ${ }^{1}$ Department of Physics, Yale University, New Haven, CT 06520-8121, USA \\ ${ }^{2}$ Physics Division, Lawrence Berkeley National Laboratory, 1 Cyclotron Road, Berkeley, CA 94720, USA \\ ${ }^{3}$ Laboratoire de Physique Nucléaire et des Hautes Energies, Université Pierre et Marie Curie, Université Paris Diderot, CNRS-IN2P3, \\ 4 Place Jussieu Tour 43, Rez de Chaussée, 75252 Paris Cedex 05, France \\ ${ }^{4}$ Université de Lyon, 69622, France \\ ${ }^{5}$ Université de Lyon 1, France \\ ${ }^{6}$ CNRS/IN2P3, Institut de Physique Nucléaire de Lyon, France \\ ${ }^{7}$ Benoziyo Center for Astrophysics, Weizmann Institute of Science, 76100 Rehovot, Israel \\ ${ }^{8}$ Department of Astronomy, Universidad de Chile, Casilla 36-D, Santiago, Chile \\ ${ }^{9}$ Department of Astronomy, California Institute of Technology, 1200 E. California Boulevard, MS 105-24, Pasadena, CA 91125, USA \\ ${ }^{10}$ Computational Cosmology Center, Lawrence Berkeley National Laboratory, 1 Cyclotron Road, Berkeley, CA 94720, USA \\ ${ }^{11}$ Centre de Recherche Astronomique de Lyon, 9 Avenue Charles André, 69561 Saint Genis Laval Cedex, France \\ ${ }^{12}$ Centre de Physique des Particules de Marseille, 163 Av. Luminy, 13288 Marseille Cedex 09, France \\ ${ }^{13}$ Center for Cosmology and Particle Physics, New York University, 4 Washington Place, New York, NY 10003 \\ Received 2009 December 15; accepted 2010 March 3; published 2010 March 30
}

\begin{abstract}
We present photometric and spectroscopic observations of SN 2007if, an overluminous $\left(M_{V}=-20.4\right)$, red $\left(B-V=0.16\right.$ at $B$-band maximum), slow-rising $\left(t_{\text {rise }}=24\right.$ days) type Ia supernova (SN Ia) in a very faint $\left(M_{g}=-14.10\right)$ host galaxy. A spectrum at 5 days past $B$-band maximum light is a direct match to the superChandrasekhar-mass candidate SN Ia 2003fg, showing Si II and C II at $\sim 9000 \mathrm{~km} \mathrm{~s}^{-1}$. A high signal-to-noise co-addition of the SN spectral time series reveals no Na I D absorption, suggesting negligible reddening in the host galaxy, and the late-time color evolution has the same slope as the Lira relation for normal SNe Ia. The ejecta appear to be well mixed, with no strong maximum in $I$ band and a diversity of iron-peak lines appearing in near-maximumlight spectra. SN 2007if also displays a plateau in the $\mathrm{Si}$ II velocity extending as late as +10 days, which we interpret as evidence for an overdense shell in the SN ejecta. We calculate the bolometric light curve of the SN and use it and the Si II velocity evolution to constrain the mass of the shell and the underlying SN ejecta, and demonstrate that SN 2007if is strongly inconsistent with a Chandrasekhar-mass scenario. Within the context of a "tamped detonation" model appropriate for double-degenerate mergers, and assuming no host extinction, we estimate the total mass of the system to be $2.4 \pm 0.2 M_{\odot}$, with $1.6 \pm 0.1 M_{\odot}$ of ${ }^{56} \mathrm{Ni}$ and with $0.3-0.5 M_{\odot}$ in the form of an envelope of unburned carbon/ oxygen. Our modeling demonstrates that the kinematics of shell entrainment provide a more efficient mechanism than incomplete nuclear burning for producing the low velocities typical of super-Chandrasekhar-mass SNe Ia.
\end{abstract}

Key words: supernovae: general - supernovae: individual (SNe 2003fg, 2006gz, 2007if, 2009dc) - white dwarfs

Online-only material: color figures

\section{INTRODUCTION}

Type Ia supernovae (SNe Ia) are of vital importance as luminosity distance indicators for measuring the expansion history of the Universe (Riess et al. 1998; Perlmutter et al. 1999). They have a small dispersion $(\sim 0.35 \mathrm{mag})$ in intrinsic peak luminosity, which can be further reduced to $0.16-0.18$ mag by applying a well-known correction dependent on the width, or decay rate, of the light curve (Phillips et al. 1999; Guy et al. 2007; Jha et al. 2007). Searches for other luminosity correlates with which to derive even more accurate luminosity distances from $\mathrm{SNe}$ Ia are underway, with some methods delivering core Hubble residual dispersions as low as 0.12 mag (Bailey et al. 2009; Wang et al. 2009; Folatelli et al. 2010). SNe Ia are generally understood to result from the thermonuclear explosion of at least one carbon/oxygen white dwarf. However, the underlying distribution of SN Ia progenitor systems and explosions mechanisms, and the relative rates of possible different physi-

\footnotetext{
${ }^{14}$ richard.scalzo@yale.edu
}

cal subclasses of SN Ia events, remain poorly constrained, with potential consequences for the average luminosity of events. Any systematic effect which may influence the luminosity of different SN Ia subpopulations at the level of a few percent has become a cause for concern for next-generation experiments, particularly redshift-dependent effects (Kim et al. 2004; Linder 2004). A better understanding of the progenitor systems will place these corrections on a much firmer conceptual footing and place limits on hitherto uncontrolled astrophysical systematics in SN Ia luminosity distance measurements.

Rare SN Ia events displaying extreme characteristics or evidence of unusual physics can often point the way toward other, less extreme instances of similar physics which may be lurking in the otherwise undifferentiated sample of "normal" events. For example, one commonly invoked rationale for the uniformity in pre-correction SN Ia luminosities is that they start with the same amount of fuel, and are triggered by the same physics: the SN Ia progenitor explodes when its mass nears the Chandrasekhar limit, $M_{\mathrm{Ch}}=1.4 M_{\odot}$, its mass is completely unbound and converted mostly to heavier elements, especially 
${ }^{56} \mathrm{Ni}$, the decay of which powers the SN Ia light curve. The single-degenerate scenario (SD; Whelan \& Iben 1973) ensures that the white dwarf slowly approaches $M_{\mathrm{Ch}}$ via accretion from a non-degenerate companion. In contrast, the double-degenerate scenario (DD; Iben \& Tutukov 1984), in which two white dwarfs in a binary system merge and explode, provides a way for SN Ia progenitors to exceed $M_{\mathrm{Ch}}$ and to give rise to more luminous events. There are also some arguments that SD, differentially rotating white dwarfs with mass exceeding $M_{\mathrm{Ch}}$ significantly can exist (Yoon \& Langer 2005), although the inclusion of baroclinic and magnetohydrodynamic instabilities appears to preclude this (e.g., Piro 2008). There may therefore be a population of $\mathrm{SNe}$ Ia with a distribution of masses greater than $M_{\mathrm{Ch}}$, with different explosion physics that interferes with luminosity standardization. The relative rate of such events among SNe Ia in general may also depend on redshift, and unless they can be identified or their luminosities accurately calibrated, they need not be common to produce significant biases in reconstructions of the dark energy equation of state.

There are at least four documented examples of overluminous SN Ia explosions with progenitor mass potentially exceeding $M_{\mathrm{Ch}}$. The first known example of the class was SN $2003 \mathrm{fg}$ (SNLS-03D3bb; Howell et al. 2006); SN 2006gz (Hicken et al. 2007), SN 2007if (Akerlof et al. 2007), and SN 2009dc (Tanaka et al. 2009; Yamanaka et al. 2009; Silverman et al. 2010) were discovered later as events spectroscopically similar to SN 2003fg. The main evidence cited for a very massive progenitor in each of these cases was the extremely high luminosity of each of these events, and by inference unusually large ${ }^{56} \mathrm{Ni}$ synthesis. SNe 2003fg, and later SNe 2006gz and 2009dc, were noted for being overluminous $\left(M_{V} \sim-20\right)$, with unusually wide light curves ("stretch" $s>1.1$ ) and CII lines, evidence for unburned carbon, in early or near-maximum spectra. In SN 2003fg, narrow, low-velocity (8000-9000 $\mathrm{km} \mathrm{s}^{-1}$ ) Si II lines near maximum light have been interpreted as evidence for a high gravitational binding energy, further supporting the hypothesis of a very massive progenitor; low velocities were also found in SN 2009dc. Ejecta velocities inferred from spectra of SN 2006gz were closer to those of normal SNe Ia.

SN 2007if was discovered by the Texas Supernova Search (Akerlof et al. 2007) in unfiltered ROTSE-IIIb images taken on 2007 August 16.3 UT. It was found independently as SNF20070825-001 by the Nearby Supernova Factory (SNfactory; Aldering et al. 2002), in an image taken in a red (RG610) filter with the QUEST-II camera (Baltay et al. 2007) on the Palomar Observatory Oschin $1.2 \mathrm{~m}$ Schmidt telescope ("PalomarQUEST") on 2007 August 25.4. No host galaxy was visible in the discovery images; nor in any available sky survey images, including Sloan Digital Sky Survey (SDSS), POSS, and USNO, making the redshift determination and interpretation of earlyphase spectra uncertain. An optical spectrum we obtained with the SuperNova Integral Field Spectrograph (SNIFS; Lantz et al. 2004) on the University of Hawaii $2.2 \mathrm{~m}$ telescope on 2007 August 26.5 UT revealed a blue continuum not obviously like a SN Ia. Spectroscopy taken at the Hobby-Eberly Telescope on 2007 August 29 also failed to identify the nature of the event (Akerlof et al. 2007). However, a later spectrum we obtained with the Double Spectrograph on the Hale $5 \mathrm{~m}$ telescope at Palomar, on 2007 September 6.5 UT, identified SN 2007if as a SN Ia, apparently well before peak. A further SNIFS spectrum taken on September 10.5 UT turned out to be an unambiguous match to a published spectrum of SN 2003fg (Howell et al. 2006). Cross-correlation of the September 10.5 SNIFS spectrum with the SNLS spectrum of SN 2003fg suggested a redshift of $0.070 \pm 0.005$.

The faintness of the host, coupled with the unusually large luminosity of the SN, presented a challenge for the detection of line emission from the host as late as a full year after explosion. More recently, however, an optical spectrum of the host galaxy was obtained on 2009 August 24.5 with the Low Resolution Imaging Spectrograph (LRIS) at the Keck Telescope on Mauna Kea, showing $\mathrm{H} \alpha$ and $\mathrm{O}$ III $\lambda 3727$ at a heliocentric redshift of $0.07416 \pm 0.00082$. This new redshift measurement allows more accurate determination of the SN luminosity and the ejecta velocity scale, which in turn enables a measurement of the total mass in the explosion.

In Sections 2 and 3, we present our detailed photometric and spectroscopic observations of SN 2007if and its host galaxy. In Section 4, we present the bolometric light curve of the SN and an estimate of the mass of ${ }^{56} \mathrm{Ni}$ synthesized in the explosion, which we find to nominally exceed $M_{\mathrm{Ch}}$. In Section 5, we argue that the red color of the $\mathrm{SN}$, its unusually long (24-day) rise time, and the existence of a plateau in the inferred photospheric velocity are best explained by the existence of an overdense shell in the ejecta, probably caused by the entrainment of an unburned carbon-oxygen envelope. We also estimate the total mass ejected in the SN, and the fraction of that mass residing in the shell and envelope. Since SN Ia mass estimates are often sensitive to the assumed kinetic energy of the explosion, we consider in Section 6 the importance of shell structure on arguments associated with mass estimates in the literature, and ask whether shell structure may be more common in superChandrasekhar-mass SN Ia candidates than previously believed. We summarize and conclude in Section 7.

\section{OBSERVATIONS}

\subsection{Optical Spectroscopy}

Observations of SN 2007if were obtained with SNIFS (Aldering et al. 2002; Lantz et al. 2004), operated by the SNfactory; the observing log is shown in Table 1. SNIFS is a fully integrated instrument optimized for automated observation of point sources on a diffuse background over the full optical window at moderate spectral resolution. It consists of a high-throughput wide-band pure-lenslet integral field spectrograph (IFS, "à la TIGER”; Bacon et al. 1995, 2000, 2001), a multifilter photometric channel to image the field surrounding the IFS for atmospheric transmission monitoring simultaneous with spectroscopy, and an acquisition/guiding channel. The IFS possesses a fully filled 6." $4 \times 6$ ". 4 spectroscopic field of view (FOV) subdivided into a grid of $15 \times 15$ spatial elements (spaxels), a dual-channel spectrograph covering 3200-5200 $\AA$ and 5100-10000 A simultaneously, and an internal calibration unit (continuum and arc lamps). SNIFS is continuously mounted on the south bent Cassegrain port of the University of Hawaii $2.2 \mathrm{~m}$ telescope (Mauna Kea) and is operated remotely. The SNIFS spectra of SN 2007if were reduced using our dedicated data reduction procedure, similar to that presented in Section 4 of Bacon et al. (2001). A brief discussion on the spectrographic pipeline was presented in Aldering et al. (2006); here we outline changes to the pipeline since that work, but leave a complete discussion of the reduction pipeline to subsequent publications focused on the instrument itself.

After standard CCD preprocessing and subtraction of a lowamplitude diffuse-light component, the 225 spectra from the individual spaxels of each SNIFS exposure were extracted from 
Table 1

Observing Log for SNIFS Spectra of SN 2007if

\begin{tabular}{crcc}
\hline \hline MJD $^{\mathrm{a}}$ & Phase $^{\mathrm{b}}$ & Exp. Time (s) & Airmass \\
\hline 54338.5 & -9.0 & 1200 & 1.004 \\
54353.5 & 4.9 & 1900 & 1.003 \\
54355.5 & 6.8 & 1230 & 1.010 \\
54358.5 & 9.6 & 900 & 1.013 \\
54363.5 & 14.2 & 1740 & 1.005 \\
54365.5 & 16.1 & 1970 & 1.003 \\
54373.4 & 23.5 & 1800 & 1.007 \\
54375.5 & 25.4 & 2000 & 1.004 \\
54378.5 & 28.2 & 1800 & 1.005 \\
54378.5 & 28.2 & 1800 & 1.025 \\
54385.4 & 34.7 & 1800 & 1.005 \\
54390.4 & 39.3 & 1800 & 1.003 \\
54393.4 & 42.1 & 2000 & 1.027 \\
54395.4 & 43.9 & 1800 & 1.034 \\
54395.4 & 43.9 & 1800 & 1.009 \\
54400.4 & 48.6 & 1800 & 1.017 \\
54400.4 & 48.6 & 1800 & 1.052 \\
54403.4 & 51.4 & 1800 & 1.017 \\
54403.4 & 51.4 & 1800 & 1.052 \\
54415.3 & 62.5 & 1800 & 1.052 \\
54420.3 & 67.2 & 2200 & 1.003 \\
\hline
\end{tabular}

Notes.

a Observer frame JD-2400000.5.

${ }^{\mathrm{b}}$ In rest-frame days relative to $B$-band maximum light.

each blue and red spectrograph exposure, and re-packed into two $(x, y, \lambda)$-datacubes. This highly specific extraction is based upon a detailed optical model of the instrument including interspectrum crosstalk corrections. The datacubes were then wavelength-calibrated, using arc lamp exposures acquired immediately after the science exposures, and spectro-spatially flatfielded, using continuum lamp exposures obtained during the same night. Cosmic rays were detected and corrected using a three-dimensional-filtering scheme applied to the datacubes.

SN and standard star spectra were extracted from each $(x, y, \lambda)$-datacube using three-dimensional point-spread function (PSF) fit photometry over a uniform background (Buton 2009). The PSF is modeled semianalytically as a constrained sum of a Gaussian (describing the core) and a Moffat function (simulating the wings). The correlations between the different shape parameters, as well as their wavelength dependencies, have been trained on a set of 300 standard star observations in various conditions of seeing and telescope focus between 2004 and 2007 with SNIFS. This resulted in a chromatic PSF empirical model depending only on an effective width (mimicking seeing) and a flattening parameter (for small imaging defocus and guiding errors). The three-dimensional PSF fit takes the atmospheric differential refraction into account without resampling.

During photometric nights, the SN spectra were flux calibrated using a flux solution and a mean atmospheric extinction derived simultaneously from all spectrophotometric standard stars observed during the same night (Buton 2009). In non-photometric conditions, an effective attenuation measurement for each exposure was made using the stars observed by the SNIFS multifilter photometric channel. Objects in the field (spatially subdivided into five regions each monitoring a different wavelength range and treated separately) were detected using SExtractor (Bertin \& Arnouts 1996), and the produced catalogs matched to (manually inspected) star catalogs created from deep stacks of the same field. An adapted version of the Supernova Legacy Survey photometry code, poloka, was then used to determine the so-called photometric flux ratio between each exposure and a reference exposure chosen from a reference night considered to be photometric. A convolution kernel is computed using the matched objects between each pair of images in order to make both comparable flux-wise, meaning that when it is applied to the best quality image (the reference one, with best seeing) it homogenizes it to the same "photometric frame" as the worst image. The photometric flux ratio equals the integral of the convolution kernel, an estimation of the relative flux extinction in each filter band for the observations on non-photometric nights, and is used to correct for attenuation by clouds. Seeing and the stability of the atmospheric transmission were assessed using quantitative analyses of SNIFS guider video frames acquired during our exposures, along with deglitched CFHT Skyprobe data.

To supplement the ANDICAM imaging photometry discussed in the next section, we synthesized additional rest-frame Bessell BVRI photometry from the SNIFS flux-calibrated spectra. The synthetic photometry is shown alongside the imaging photometry in Table 2 and in Figures 6 and 7.

\subsection{ANDICAM BVRI Photometry}

Follow-up BVRI photometry of SN 2007if using ANDICAM on the CTIO $1.3 \mathrm{~m}$ was obtained through the Small and Moderate Aperture Research Telescope System (SMARTS) Consortium, from 2007 September 10 through 2007 December 20. Each band was exposed for approximately $240 \mathrm{~s}$. Further spectroscopic follow-up was also obtained with SNIFS extending through 2007 November 26. Since the nature and type of SN 2007if were not immediately apparent, SMARTS observations began only on 2007 September 10.5, when the light curve was already in decline. Final reference images for subtraction of host galaxy light were obtained in the 2008 observing season; although the host galaxy is very faint, at late times its contribution could still be significant.

The SMARTS photometry was processed using an automated pipeline based on IRAF (Tody 1993). SMARTS images were bias-subtracted, overscan-subtracted, and flat-fielded using ccdproc. Point sources were detected (daofind) and their instrumental magnitudes measured (phot), then aperturecorrected to a $6 "$ aperture (mkapfile). The images were registered to a standard detector coordinate system (xyxymatch, geomap, gregister). The resampled final reference images were combined (imsum) for added depth in each band, rejecting detector defects and cosmic rays by discarding the brightest and faintest value at each pixel location in the stack. An absolute calibration (zeropoint, extinction, and color terms) was established on photometric nights from observations of Landolt (1992) standards, fitting a zeropoint and extinction coefficient for each night separately as well as a color term constant across all nights. The calibration was transferred to the field stars for each photometric night separately using the zeropoint and extinction but ignoring the color terms, producing magnitudes on a "natural" ANDICAM system which agrees with the Landolt system for stars with $B-V=V-R=R-I=0$. These calibrated magnitudes were then averaged over photometric nights to produce final calibrated ANDICAM magnitudes for the field stars. The final reference co-add was normalized to each image in turn, with the best-seeing image in each pair convolved to match the worst (fitpsf,psfmatch); the (undetected) host galaxy light was then removed by subtraction. The positions of 
Table 2

Rest-frame BVRIJ Light Curve of SN 2007if

\begin{tabular}{|c|c|c|c|c|c|c|c|}
\hline $\mathrm{MJD}^{\mathrm{a}}$ & Phase $^{\mathrm{b}}$ & $B$ & $V$ & $R$ & $I$ & $J$ & Instrument \\
\hline 54337.3 & -10.3 & $\ldots$ & $\ldots$ & $17.78 \pm 0.10$ & $\cdots$ & $\cdots$ & QUEST \\
\hline 54338.5 & -9.2 & $\cdots$ & $17.67 \pm 0.02$ & $\cdots$ & $\cdots$ & $\ldots$ & SNIFS-P \\
\hline 54338.5 & -9.2 & $17.54 \pm 0.04$ & $17.62 \pm 0.06$ & $17.53 \pm 0.04$ & $17.64 \pm 0.06$ & $\cdots$ & SNIFS-S \\
\hline 54346.3 & -2.0 & $\cdots$ & $\cdots$ & $17.15 \pm 0.07$ & $\cdots$ & $\cdots$ & QUEST \\
\hline 54353.4 & 4.6 & $\cdots$ & $\cdots$ & $17.29 \pm 0.05$ & $\cdots$ & $\cdots$ & QUEST \\
\hline 54353.5 & 4.7 & $17.45 \pm 0.04$ & $17.29 \pm 0.05$ & $17.27 \pm 0.04$ & $17.46 \pm 0.05$ & $\cdots$ & SNIFS-S \\
\hline 54354.4 & 5.5 & $17.49 \pm 0.02$ & $17.28 \pm 0.02$ & $17.28 \pm 0.01$ & $17.40 \pm 0.02$ & $\cdots$ & SMARTS \\
\hline 54354.4 & 5.6 & $\ldots$ & $\ldots$ & $17.19 \pm 0.11$ & $\ldots$ & $\cdots$ & QUEST \\
\hline 54355.5 & 6.6 & $\ldots$ & $17.29 \pm 0.02$ & $\ldots$ & $\ldots$ & $\ldots$ & SNIFS-P \\
\hline 54355.5 & 6.6 & $17.49 \pm 0.04$ & $17.24 \pm 0.04$ & $17.21 \pm 0.03$ & $17.38 \pm 0.05$ & $\ldots$ & SNIFS-S \\
\hline 54356.3 & 7.3 & $17.57 \pm 0.03$ & $17.27 \pm 0.02$ & $17.29 \pm 0.01$ & $17.43 \pm 0.02$ & $18.41 \pm 0.17$ & SMARTS \\
\hline 54358.2 & 9.2 & $17.69 \pm 0.04$ & $17.33 \pm 0.03$ & $17.34 \pm 0.01$ & $17.49 \pm 0.02$ & $18.43 \pm 0.17$ & SMARTS \\
\hline 54358.5 & 9.4 & $\ldots$ & $17.36 \pm 0.02$ & $\ldots$ & $\ldots$ & $\ldots$ & SNIFS-P \\
\hline 54358.5 & 9.4 & $17.66 \pm 0.09$ & $17.19 \pm 0.09$ & $17.18 \pm 0.05$ & $17.34 \pm 0.10$ & $\cdots$ & SNIFS-S \\
\hline 54361.3 & 12.0 & $17.96 \pm 0.02$ & $17.50 \pm 0.03$ & $17.47 \pm 0.01$ & $17.58 \pm 0.03$ & $\cdots$ & SMARTS \\
\hline 54363.2 & 13.8 & $18.17 \pm 0.03$ & $17.55 \pm 0.03$ & $17.47 \pm 0.04$ & $17.60 \pm 0.03$ & $19.16 \pm 0.14$ & SMARTS \\
\hline 54363.5 & 14.0 & $\cdots$ & $17.59 \pm 0.04$ & $\cdots$ & $\cdots$ & $\cdots$ & SNIFS-P \\
\hline 54363.5 & 14.0 & $18.14 \pm 0.07$ & $17.55 \pm 0.06$ & $17.48 \pm 0.04$ & $17.59 \pm 0.07$ & $\cdots$ & SNIFS-S \\
\hline 54365.5 & 15.9 & $18.29 \pm 0.09$ & $17.55 \pm 0.06$ & $17.46 \pm 0.04$ & $17.52 \pm 0.07$ & $\cdots$ & SNIFS-S \\
\hline 54366.3 & 16.6 & $18.52 \pm 0.06$ & $17.71 \pm 0.05$ & $17.62 \pm 0.05$ & $17.68 \pm 0.11$ & $\cdots$ & SMARTS \\
\hline 54372.3 & 22.2 & $19.05 \pm 0.32$ & $\cdots$ & $17.68 \pm 0.23$ & $17.55 \pm 0.29$ & $19.08 \pm 0.13$ & SMARTS \\
\hline 54373.4 & 23.3 & $19.02 \pm 0.12$ & $18.06 \pm 0.08$ & $17.76 \pm 0.05$ & $17.63 \pm 0.06$ & $\cdots$ & SNIFS-S \\
\hline 54375.2 & 24.9 & $19.38 \pm 0.08$ & $18.22 \pm 0.04$ & $17.85 \pm 0.02$ & $17.73 \pm 0.04$ & $19.17 \pm 0.20$ & SMARTS \\
\hline 54375.4 & 25.2 & $19.15 \pm 0.11$ & $18.16 \pm 0.06$ & $17.85 \pm 0.04$ & $17.73 \pm 0.05$ & $\cdots$ & SNIFS-S \\
\hline 54378.2 & 27.8 & $19.49 \pm 0.06$ & $18.37 \pm 0.03$ & $17.98 \pm 0.02$ & $17.85 \pm 0.03$ & $\ldots$ & SMARTS \\
\hline 54378.5 & 28.0 & $19.38 \pm 0.17$ & $18.33 \pm 0.09$ & $17.99 \pm 0.04$ & $17.83 \pm 0.07$ & $\cdots$ & SNIFS-S \\
\hline 54382.3 & 31.5 & $19.65 \pm 0.07$ & $18.46 \pm 0.03$ & $18.11 \pm 0.02$ & $17.85 \pm 0.03$ & $\cdots$ & SMARTS \\
\hline 54385.4 & 34.4 & $19.57 \pm 0.14$ & $18.48 \pm 0.07$ & $18.11 \pm 0.04$ & $17.87 \pm 0.06$ & $\ldots$ & SNIFS-S \\
\hline 54388.1 & 37.0 & $19.81 \pm 0.07$ & $18.62 \pm 0.05$ & $18.25 \pm 0.03$ & $17.99 \pm 0.03$ & $19.16 \pm 0.10$ & SMARTS \\
\hline 54388.2 & 37.1 & $\cdots$ & $18.59 \pm 0.07$ & $\cdots$ & $\cdots$ & $\cdots$ & SNIFS-P \\
\hline 54388.4 & 37.3 & $\cdots$ & $18.64 \pm 0.07$ & $\ldots$ & $\ldots$ & $\cdots$ & SNIFS-P \\
\hline 54388.4 & 37.3 & $19.84 \pm 0.43$ & $18.69 \pm 0.22$ & $18.26 \pm 0.10$ & $17.98 \pm 0.14$ & $\cdots$ & SNIFS-S \\
\hline 54390.4 & 39.1 & $\ldots$ & $18.71 \pm 0.05$ & $\cdots$ & $\cdots$ & $\cdots$ & SNIFS-P \\
\hline 54390.4 & 39.1 & $19.70 \pm 0.15$ & $18.66 \pm 0.09$ & $18.31 \pm 0.05$ & $18.05 \pm 0.07$ & $\cdots$ & SNIFS-S \\
\hline 54392.2 & 40.8 & $19.88 \pm 0.10$ & $18.81 \pm 0.04$ & $18.38 \pm 0.03$ & $18.14 \pm 0.04$ & $\cdots$ & SMARTS \\
\hline 54393.3 & 41.8 & $\ldots$ & $18.81 \pm 0.05$ & $\ldots$ & $\ldots$ & $\cdots$ & SNIFS-P \\
\hline 54393.4 & 41.9 & $19.79 \pm 0.26$ & $18.77 \pm 0.14$ & $18.39 \pm 0.07$ & $18.13 \pm 0.10$ & $\cdots$ & SNIFS-S \\
\hline 54395.1 & 43.5 & $19.88 \pm 0.23$ & $\cdots$ & $\cdots$ & $18.45 \pm 0.13$ & $\cdots$ & SMARTS \\
\hline 54395.3 & 43.7 & $19.83 \pm 0.18$ & $18.82 \pm 0.10$ & $18.48 \pm 0.06$ & $18.22 \pm 0.07$ & $\cdots$ & SNIFS-S \\
\hline 54400.2 & 48.2 & $20.49 \pm 0.49$ & $\cdots$ & $18.68 \pm 0.25$ & $18.38 \pm 0.25$ & $20.55 \pm 0.27$ & SMARTS \\
\hline 54400.4 & 48.4 & $\cdots$ & $19.06 \pm 0.09$ & $\cdots$ & $\ldots$ & $\cdots$ & SNIFS-P \\
\hline 54400.4 & 48.4 & $19.74 \pm 0.51$ & $19.19 \pm 0.21$ & $18.90 \pm 0.11$ & $18.71 \pm 0.13$ & $\cdots$ & SNIFS-S \\
\hline 54403.4 & 51.2 & $\ldots$ & $19.09 \pm 0.04$ & $\cdots$ & $\cdots$ & $\cdots$ & SNIFS-P \\
\hline 54403.4 & 51.2 & $20.07 \pm 0.51$ & $19.14 \pm 0.22$ & $18.83 \pm 0.13$ & $18.60 \pm 0.16$ & $\ldots$ & SNIFS-S \\
\hline 54404.2 & 52.0 & $20.04 \pm 0.10$ & $19.18 \pm 0.06$ & $18.75 \pm 0.04$ & $18.61 \pm 0.06$ & $20.43 \pm 0.26$ & SMARTS \\
\hline 54408.2 & 55.6 & $20.20 \pm 0.10$ & $19.26 \pm 0.05$ & $18.94 \pm 0.04$ & $18.61 \pm 0.05$ & $20.77 \pm 0.33$ & SMARTS \\
\hline 54412.3 & 59.5 & $20.20 \pm 0.10$ & $19.36 \pm 0.05$ & $19.08 \pm 0.04$ & $18.97 \pm 0.07$ & $20.99 \pm 0.45$ & SMARTS \\
\hline 54415.3 & 62.3 & $\ldots$ & $19.28 \pm 0.05$ & $\ldots$ & $\ldots$ & $\ldots$ & SNIFS-P \\
\hline 54415.3 & 62.3 & $20.31 \pm 0.33$ & $19.43 \pm 0.20$ & $19.19 \pm 0.12$ & $18.98 \pm 0.17$ & $\ldots$ & SNIFS-S \\
\hline 54418.1 & 64.9 & $20.28 \pm 0.10$ & $19.50 \pm 0.05$ & $19.23 \pm 0.04$ & $19.22 \pm 0.07$ & $\cdots$ & SMARTS \\
\hline 54420.3 & 66.9 & & $19.50 \pm 0.04$ & $\cdots$ & $\cdots$ & $\cdots$ & SNIFS-P \\
\hline 54420.3 & 67.0 & $20.25 \pm 0.22$ & $19.42 \pm 0.14$ & $19.25 \pm 0.09$ & $19.09 \pm 0.12$ & $\cdots$ & SNIFS-S \\
\hline 54422.1 & 68.6 & $20.16 \pm 0.14$ & $19.50 \pm 0.08$ & $19.41 \pm 0.06$ & $19.26 \pm 0.09$ & $\cdots$ & SMARTS \\
\hline 54430.4 & 76.4 & $\cdots$ & $19.49 \pm 0.10$ & $\cdots$ & $\cdots$ & $\cdots$ & SNIFS-P \\
\hline 54431.1 & 77.0 & $20.40 \pm 0.27$ & $19.60 \pm 0.14$ & $19.65 \pm 0.14$ & $19.38 \pm 0.13$ & $\ldots$ & SMARTS \\
\hline 54435.1 & 80.7 & $20.67 \pm 0.17$ & $19.76 \pm 0.07$ & $19.69 \pm 0.07$ & $19.63 \pm 0.15$ & $\cdots$ & SMARTS \\
\hline 54438.1 & 83.5 & $20.70 \pm 0.15$ & $19.73 \pm 0.06$ & $19.80 \pm 0.06$ & $19.74 \pm 0.12$ & $\cdots$ & SMARTS \\
\hline 54442.1 & 87.2 & $20.53 \pm 0.14$ & $19.82 \pm 0.07$ & $19.96 \pm 0.08$ & $20.23 \pm 0.23$ & $\cdots$ & SMARTS \\
\hline 54445.1 & 90.0 & $20.84 \pm 0.18$ & $20.00 \pm 0.08$ & $19.81 \pm 0.07$ & $19.81 \pm 0.14$ & $\cdots$ & SMARTS \\
\hline 54448.1 & 92.8 & $20.87 \pm 0.18$ & $19.92 \pm 0.07$ & $19.98 \pm 0.07$ & $19.88 \pm 0.14$ & $\cdots$ & SMARTS \\
\hline 54451.1 & 95.6 & $20.84 \pm 0.33$ & $19.82 \pm 0.12$ & $20.00 \pm 0.14$ & $19.79 \pm 0.18$ & $\cdots$ & SMARTS \\
\hline
\end{tabular}

Notes.

a Observer frame J $D-2400000.5$.

${ }^{\mathrm{b}}$ In rest-frame days relative to $B$-band maximum light. 
all detections of the $\mathrm{SN}$ with signal-to-noise ratio $(\mathrm{S} / \mathrm{N})$ greater than 10 were averaged, and this final position used to measure the flux in each image, in order to achieve more accurate photometry for noisy measurements at late times. Systematic errors were estimated for the resampling, flux normalization and PSF convolution steps by measuring the dispersion in the residuals (from a light curve constant in time) of the field stars. We estimate an end-to-end systematic floor of $1 \%$ on our differential photometry, so that our observations of the SN are limited only by sky noise.

To characterize the ANDICAM system throughput as a function of wavelength in each band, we use a product in each band of the KPNO filter transmission curve, the quantum efficiency of a Fairchild $447 \mathrm{CCD}$ as measured by the manufacturer (including $\mathrm{MgO}_{2}$ quartz window transmission), and the reflectivity of two layers of aluminum. We adjust the central wavelength of each ANDICAM passband using SMARTS observations of spectrophotometric standards (Stritzinger et al. 2005), such that our calibrated natural-system photometry matches synthetic photometry in the adjusted passbands from the standard star spectra. After these steps, the SN's ANDICAM-system BVRI light curve was measured by comparison to field stars. The observer-frame ANDICAM magnitudes were corrected for Galactic extinction using $E(B-V)=0.079$ (Schlegel et al. 1998) and $R_{V}=3.1$ (Cardelli et al. 1988), then $K$-corrected (Nugent et al. 2002) to rest-frame Bessell BVRI bandpasses (Bessell 1990), using the adjusted ANDICAM filter bandpasses and the SNIFS spectral time series.

\subsection{ANDICAM J Photometry}

The SMARTS observations were taken with ANDICAM in dual CCD-IR mode, so that a $J$ light curve was obtained simultaneously with the BVRI light curve. Four dithered $50 \mathrm{~s}$ $J$-band exposures were taken during each $240 \mathrm{~s}$ optical-band exposure, for a total $J$ exposure time of $800 \mathrm{~s}$ each night.

The $J$-band images were reduced using IRAF. Separate bias and overscan levels for each of the four amplifiers of the detector were subtracted from the ANDICAM images, which were then flat-fielded using a superflat made from dome flat images taken nightly. Bad pixels were identified as $3 \sigma$-deviant pixels in the superflat. Sources in the field were generally detected at low $\mathrm{S} / \mathrm{N}$, precluding automatic registration of single dithered exposures. We therefore performed an initial co-add of the exposures at each dither position without further registration; we estimate a positional error of approximately 0.5 , resulting in a modest loss in $\mathrm{S} / \mathrm{N}$. Sources were then detected in the co-add at each dither position, and the single-dither co-adds were registered to each other to produce a final co-add for the night. Host galaxy subtraction was neglected for $J$ band, since registration was difficult and since the high sky noise in these measurements dominates over any systematic error from unsubtracted light from such a faint host. The field star magnitudes were calibrated using standard star observations on nights reported as photometric by the SMARTS queue observer, with extinction corrections based on a seasonal average $J$-band extinction coefficient (0.1 mag airmass ${ }^{-1}$; Frogel 1998) for CTIO. The SN magnitudes were then measured via differential photometry using three suitable non-variable stars in the FOV, similar to the process used in reducing the optical data.

While we have no near-infrared spectra of SN 2007if with which to perform $K$-corrections on the $J$-band magnitudes, we use the spectra of the 1991T-like SN 1999ee (Hamuy et al. 2002) as a reasonable approximation. The transfer function used was the product of the quantum efficiency of the Rockwell $\mathrm{HgCdTe}$ "Hawaii" array and the $J$-band filter transmission as measured by the manufacturer.

Despite this somewhat approximate treatment, we expect based on residuals for the field stars that our systematic errors are of order $10 \%$ or less, in general comparable to or less than the sky noise. These are included in our stated error bars.

\subsection{Additional Optical Photometry}

To further constrain the SN's multi-band behavior near maximum light, the ANDICAM data were supplemented with observations near maximum from the broadband RG610 data collected by the Palomar-QUEST supernova search, and with additional $V$-band photometric observations taken with the SNIFS imaging channel when adjusting the telescope's pointing to place the SN into the FOV of the SNIFS integral field unit. Stars in the RG610 images of the SN were matched to corresponding $R$-band and $I$-band images taken with ANDICAM, and their $R$ magnitudes were corrected to a natural Palomar-QUEST RG610 system using a linear color correction, $R G 610=$ $R+c(R-I)$ (so that $R G 610=R$ for a star with $R-I=0$ ). The $R G 610$ magnitudes of the $\mathrm{SN}$ in the search observations were established via comparison with several local field stars. Finally, the SN $R G 610$ magnitudes were $K$-corrected to ANDICAM $R$, using $K$-corrections calculated from the measured transmission curves for the ANDICAM $R$ and Palomar-QUEST $R G 610$ applied to SNIFS spectra on either side of maximum light. The process has an estimated error floor of about 0.05 mag. A similar procedure was followed for the SNIFS $V$-band photometry. The $R G 610$ and SNIFS $V$ photometry have been merged, respectively, with the ANDICAM $R$ and $V$ light curve points in the analysis to follow.

Finally, Akerlof et al. (2007) and F. Yuan et al. (2010, in preparation) present unfiltered photometry obtained with ROTSE-IIIb that is valuable in constraining the rise time (see Section 4.2).

\subsection{Host Galaxy Observations}

The host of SN 2007if is not visible in pre-explosion survey images, and no host spectroscopic features were detected in SNIFS spectra obtained while the SN was being actively followed. On 2009 August 24.6 UTC, imaging obtained with the LRIS (Oke et al. 1995) on the Keck-I 10 m telescope revealed an extended source coincidence with the original SN location. Five exposures each of $100 \mathrm{~s}$ duration were obtained using the LRIS blue-side camera equipped with a $g$-band filter. The exposures were dithered to allow rejection of cosmetic defects. The raw images were overscan-subtracted, flat-fielded using dome flats, astrometrically calibrated using WCSTools (Mink 2006), and then co-added using SWarp (Bertin et al. 2002). The final image, shown in Figure 1, was segmented using SExtractor (Bertin \& Arnouts 1996) and then flux calibrated using stars overlapping with SDSS-I, matched using WESIX. ${ }^{15}$ The resulting host location is $\alpha=01: 10: 51.412$, $\delta=15: 27: 39.57$ and the magnitude based on SExtractor mag_iso_cor after correcting for Galactic extinction of $A_{g}=$ 0.34 is $g=22.89 \pm 0.04$. The host major and minor axes are $1^{\prime \prime .5}$ and 1".2, respectively, uncorrected for seeing of 0.76 FWHM.

Upon detection of the host, LRIS was reconfigured for spectroscopic observations intended to measure the host redshift.

\footnotetext{
15 http://nvogre.astro.washington.edu:8080/wesix/
} 


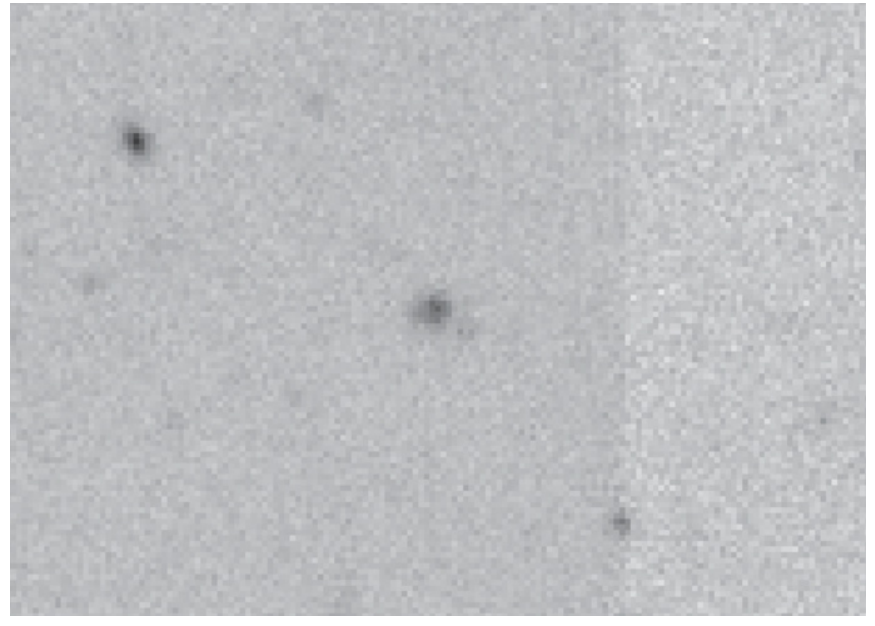

Figure 1. Image of the host galaxy of SN 2007if as co-added from Keck LRIS imaging (north is up, east is left). The FOV is $43^{\prime \prime} \times 30^{\prime \prime}$.

On the blue side the $6001 \mathrm{~mm}^{-1}$ grism blazed at $4000 \AA$ was employed, covering 3500-5600 $\AA$, while on the red side the $9001 \mathrm{~mm}^{-1}$ grating blazed at $5500 \AA$ was used to cover 5440-7640 A. The D5600 dichroic was used to direct light to the appropriate channel. A slit width of $1^{\prime \prime}$ was used, giving resolutions of $\lambda / \Delta \lambda \sim 1200$ and $\sim 1650$ for the blue and red sides, respectively. Four exposures of $900 \mathrm{~s}$ each were obtained. These were overscan-subtracted, flat-fielded using internal quartz flats, cosmic-ray rejected using LAcospec (van Dokkum 2001), and then wavelength-calibrated using arc and night sky lines. The reduced spectrum revealed weak emission lines from $\mathrm{H} \alpha$ and $\mathrm{O}$ II $\lambda 3727$. The resulting heliocentric redshift is $z=0.07416 \pm 0.00082$, and the resulting host luminosity is $M_{g}=-14.10 \pm 0.07$ after correction for Galactic extinction.

\section{DISCUSSION}

Our extensive photometric and spectroscopic data set allows us to examine several unique features of SN 2007if. We begin with comparison to the spectrum of the prototype- $\mathrm{SN}$ $2003 \mathrm{fg}$ - as well as of SN 2006gz, focusing in particular on the evidence for unburned carbon in such systems. The velocity evolution is examined, especially in light of the low velocities previously found for SN 2003fg and SN 2009dc. We then examine the photometric properties and the influence of dust extinction, in preparation for determining the mass of the $\mathrm{SN}$ 2007if progenitor.

\subsection{Spectral Features and Velocity Evolution}

Figure 2 displays selected SNIFS spectra of SN 2007if, along with selected published spectra from SN 2006gz and the only spectrum of SN 2003fg available. The SNIFS spectra of SN 2007 if are supplemented by the $+1 \mathrm{~d}$ spectrum obtained at the Palomar Hale $5.1 \mathrm{~m}$ telescope with the Double Spectrograph. The spectral time-series of SN 2006gz published by Hicken et al. (2007) extends from $-14 \mathrm{~d}$ to $+11 \mathrm{~d}$ with respect to maximum-the earliest spectrum from that event had more pronounced absorption features clearly identifying it as a SN Ia, and also a prominent $\mathrm{C}_{\text {II }} \lambda 6580$ absorption initially attributed to a low velocity Si II component (Prieto \& Depoy 2006). In contrast, the earliest SNIFS spectrum of SN 2007if is relatively featureless, and without prior knowledge of the host redshift, not obviously from a SN Ia. In general, the absorption features common to both SN 2006gz and SN 2007 if appear to be broader,

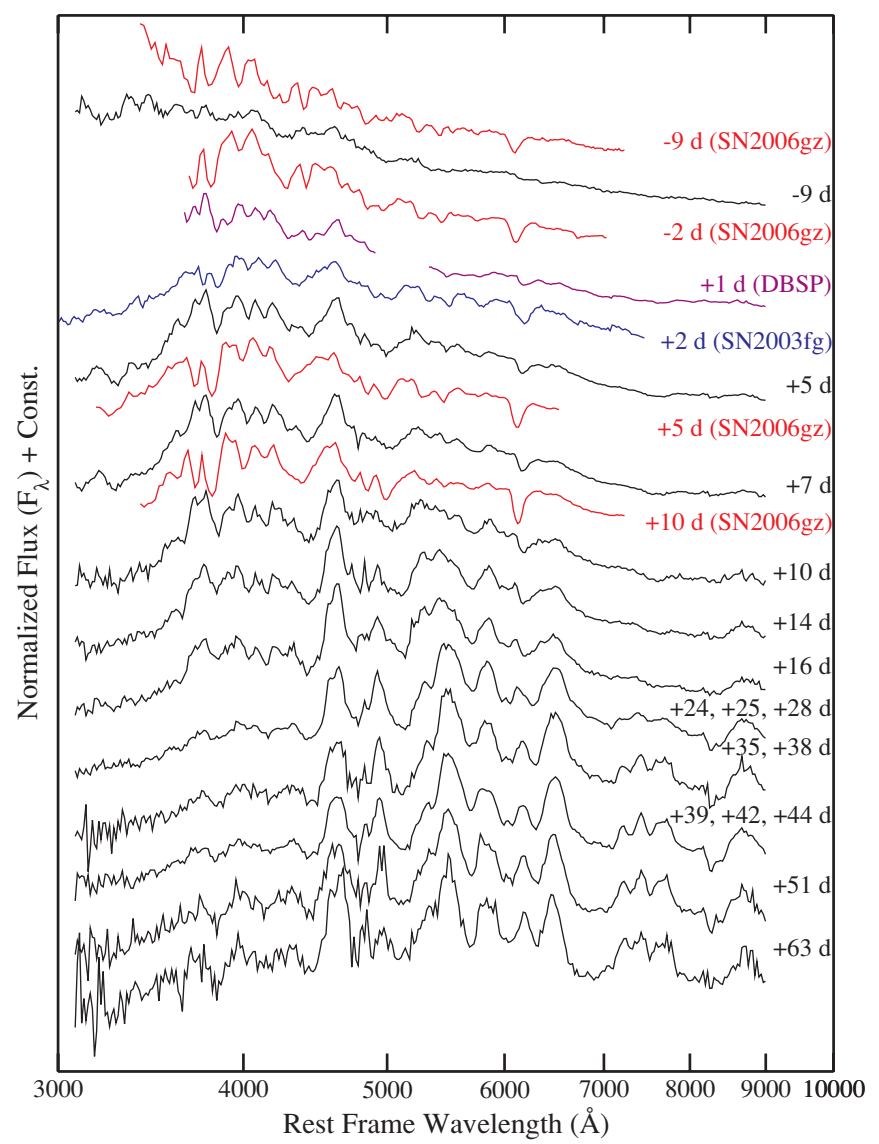

Figure 2. Selected rest-frame SNIFS spectra of SN 2007if (black curves), interleaved with observations of SNe 2006gz (red; Hicken et al. 2007) and 2003 fg (blue; the prototypical super-Chandrasekhar SN Ia candidate; Howell et al. 2006). A spectrum obtained just after maximum with the Double Spectrograph (DBSP, violet) on the $5.1 \mathrm{~m}$ Palomar Hale telescope is also included. Spectra of SN 2006gz are selected based on proximity in rest-frame phase for comparison. The $-9 \mathrm{~d}$ spectrum of SN 2006gz has more developed absorption features than SN 2007if at the same phase, making SN 2007if more difficult to recognize as a SN Ia. SN 2007if is in some respects a better match to SN 2003fg than SN 2006gz, but not in every case. Note especially the more blueshifted absorptions of SN 2006gz (especially Si II around $6100 \AA$ ). All spectra shown have been binned to $1000 \mathrm{~km} \mathrm{~s}^{-1}$ and later phase SNIFS spectra combined for presentation purposes.

(A color version of this figure is available in the online journal.)

stronger, and more highly blue-shifted in the former than in the latter-note the faster Si II absorption around 6100-6200 in SN 2006gz. Around maximum, the spectrum of SN 2007if seems to more closely resemble that of SN 2003fg than it does SN 2006gz, in particular at the double-notch absorptions around $4000-4200 \AA$, but also note the double absorption at 3700-3900 ̊̊ missing from SN 2007if. Comparison at much later phases to SN 2006gz is not possible since that time-series ends much earlier than that from SNIFS. The detailed evolution at these phases is rather slow, but these spectra appear quite similar to those of a normal SN Ia except for the lack of emission around $4000 \AA$.

In Figure 3, we present the detailed evolution of the SNIFS spectra in the region of the Si II $\lambda 6355$ feature. In the earliest spectrum, the feature with the blue absorption edge at $6360 \AA$ may be a sign of C II $\lambda 6580$ at a velocity of $\sim 10,000 \mathrm{~km} \mathrm{~s}^{-1}-$ suggesting the presence of unburned carbon as in SNe 2006gz and $2009 \mathrm{dc}$, though the line is much weaker. Due to the redshift of SN 2007if, this region overlaps telluric $B$-band absorption, but we have confirmed the accuracy of our telluric 


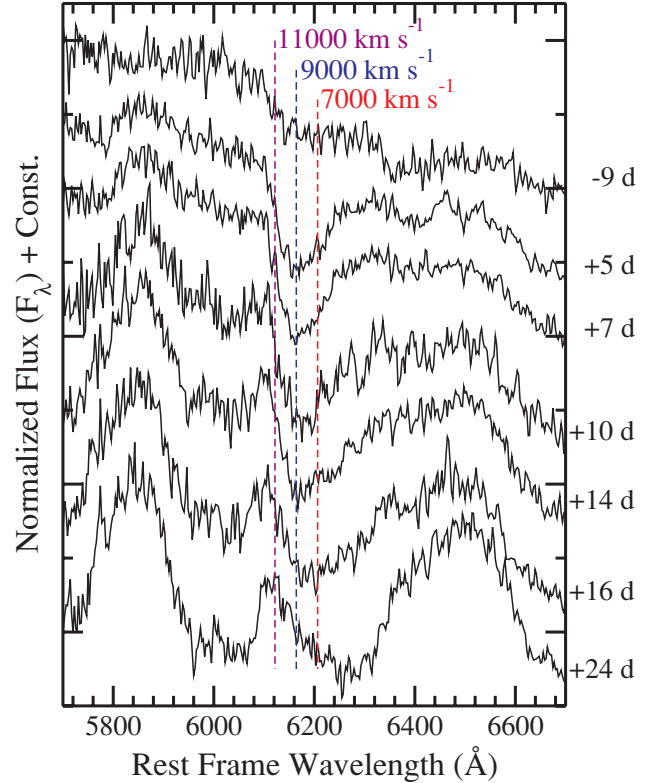

Figure 3. Detailed evolution of SN 2007if spectra around the Si II $\lambda 6355$ feature. (A color version of this figure is available in the online journal.)

correction and believe the notch to be a real SN feature. The evidence for $\mathrm{C}_{\mathrm{II}}$ at this wavelength in SN 2003fg is more circumstantial owing to that spectrum's lower $\mathrm{S} / \mathrm{N}$ at these wavelengths and the putative identification of $\mathrm{C}_{\text {II }} \lambda 4267$ (but see below). Immediately blueward is the expected position of Si II $\lambda 6355$, at which an absorption "slump" is detectable. The sloping continuum and proximity to $\mathrm{C}_{\text {II }}$ make the line profile difficult to measure directly; we factor out the continuum in our measurements of the absorption minimum described below, and include related uncertainties in our error bars. In any case, the shallow inflection near $9000 \mathrm{~km} \mathrm{~s}^{-1}$ and the blue edge near $11,000 \mathrm{~km} \mathrm{~s}^{-1}$ (both relative to $6355 \AA$ ), coincident with the edge of the Si II feature in the post-maximum spectra, suggest that the photosphere may have receded to the post-maximum velocity at a phase of $-9 \mathrm{~d}$. The relative robustness of this edge across phase may suggest that the layer of Si-peak elements production extends only to $11,000 \mathrm{~km} \mathrm{~s}^{-1}$.

Figure 4 compares the evolution of the position of the Si II $\lambda 6355$ absorption minimum in SNe 2007if, 2006gz, 2009dc, and 2003fg. At least in the case of SN 2007if, by the time of the next SNIFS observation after day +16 (on day +24 ), the $\mathrm{SN}$ spectrum has evolved too much to associate Si II $\lambda 6355$ with any given spectral feature without peril. We exclude the DBSP spectrum from this analysis, since it was obtained at lower resolution and in poor conditions; however a measurement from the profile is consistent with the SNIFS observations a few days later. The measurements of Si II $\lambda 6355$ on SNIFS spectra were made as follows: bins in each spectrum immediately to the right and left of the line feature were used to fit a linear local continuum, $F_{\lambda, \text { cont }}=a+b \lambda$. The spectrum in the region of the line was then fitted using $F_{\lambda}=F_{\lambda \text {,cont }} \times \phi(\lambda)$ where the absorption line was modeled as a skewed Gaussian,

$$
\phi(\lambda)=1-c\left[1+d\left(\lambda-\lambda_{0}\right)^{3}\right] e^{-\left(\lambda-\lambda_{0}\right)^{2} / 2 \sigma^{2}} .
$$

The line velocity was determined by solving $d \phi / d \lambda=0$ via the Newton-Raphson method. The error bars on the procedure were determined through a bootstrap Monte Carlo in two stages. In the first stage, values of $a$ and $b$ representing possible

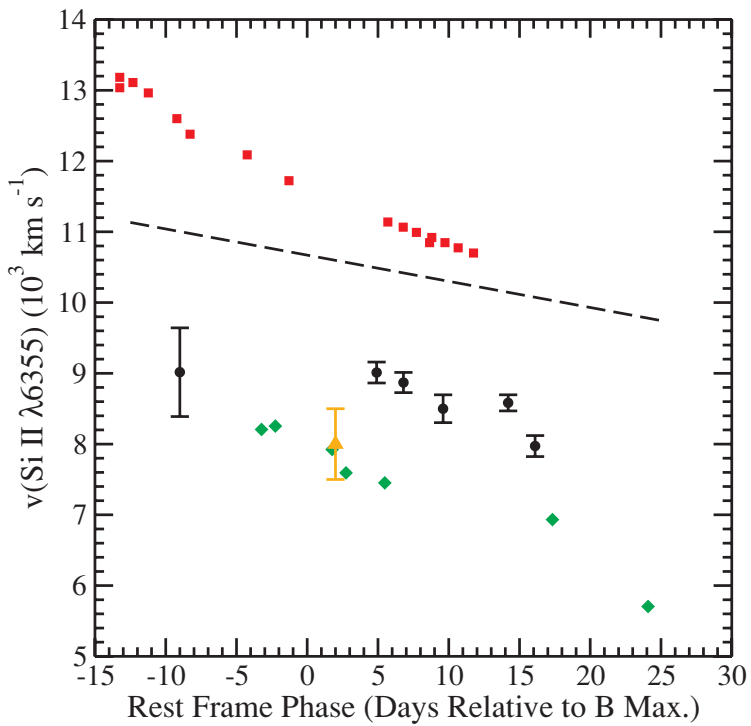

Figure 4. Comparison of the evolution of the Si II absorption feature in SN 2007if (black circles) with SNe 2006gz (red squares), 2009dc (green diamonds), and 2003 fg (orange triangle). The mean evolution of Si II velocity in normal "LVG" subclass SNe Ia (Benetti et al. 2005) is presented as the dashed line.

(A color version of this figure is available in the online journal.)

continua $F_{\lambda \text {,cont }}$ were sampled using the covariance matrix of the local continuum fit; for each candidate continuum, the line profile $\phi(\lambda)$ was then refit holding $a$ and $b$ fixed, and values of $c, d, \lambda_{0}$, and $\sigma$ were sampled using the covariance matrix of the fit to $\phi(\lambda)$. The final velocity values and their errors were determined as the mean and standard deviation of the distribution of absorption minimum velocities thus generated. We can see in Figure 4 the measurements of the velocity and their error with this method for each spectrum of the study. Clearly, the average measured characteristic ejecta velocities of SNe 2007if and 2009dc are much more consistent with that measured for SN $2003 \mathrm{fg}$, the prototypical super-Chandrasekhar event. The shallow slope of the velocity evolution $(\dot{v}=34 \pm$ $15 \mathrm{~km} \mathrm{~s}^{-1}$ day $^{-1}$ ) is characteristic of the low-velocity-gradient (LVG; Benetti et al. 2005) subclass.

To further characterize the SN ejecta, we have analyzed the +5 day SNIFS spectrum using the automated SN spectroscopic direct analysis program, synapps (Thomas et al. 2009). This code incorporates the familiar SYNOW-style (Jeffery \& Branch 1990; Branch et al. 2003) parameterized model as part of an objective function in a multidimensional nonlinear optimization problem. From a good fit the presence of given ions may be ruled out, and for ions that are positively identified, corresponding intervals of ejecta velocity are constrained. Such a parameterized approach is indispensable in the analysis of such particularly unusual events as SN 2007if for which no reasonable detailed theoretical prediction otherwise exists, and will continue to play a vital role as long as new types of transients are discovered.

A fit to the day +5 spectrum appears in Figure 5. The entire SNIFS wavelength range is included in the fit. Familiar SN Ia ion species Mg II, Si II, S II, Ca II, Fe II and Fe III produce unambiguous spectroscopic absorption signatures. Blanketing by iron-peak elements is invoked to suppress UV flux, but the optimized model may overcompensate for the tendency of the Sobolev approximation to underblanket here. The Si II and $\mathrm{S}$ II features are rather weak, a trait in common with the socalled overluminous or SN 1991T-like SNe Ia. The Ca II H\&K absorption at $3800 \AA$ is extremely sharp and weak compared to 


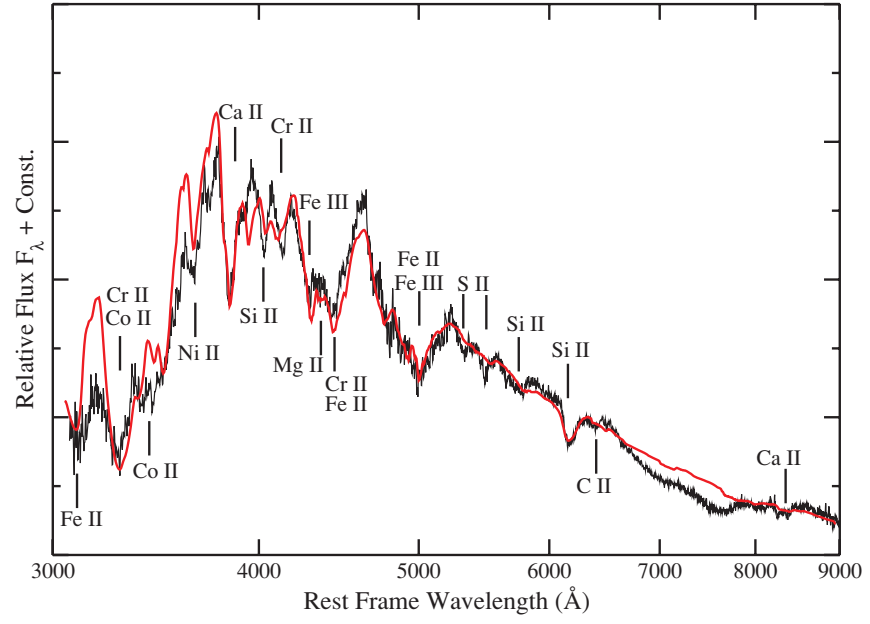

Figure 5. SYNOW fit to the SNIFS day +5 spectrum.

(A color version of this figure is available in the online journal.)

normal events, as was noted in the case of SN 2003fg. The highly blended regions centered on $4500 \AA$ and $5000 \AA$ are actually dominated by Fe III, again harkening to SN 1991T-like events. The relatively weak IME signatures and the prevalence of Fe III indicate high temperatures in the ejecta; in SN 1991T this has been attributed to the presence of ${ }^{56} \mathrm{Ni}$ in the outer layers of the ejecta (Mazzali et al. 1995). We also detect a weak signature of C II at around $8000 \mathrm{~km} \mathrm{~s}^{-1}$, i.e., at velocities comparable to the Si II feature.

Of particular interest is the pair of sharp notches at $4030 \AA$ and $4130 \AA$. In Howell et al. (2006), the bluer notch is attributed to $\mathrm{Si}$ II, and the redder to $\mathrm{C}$ II. In the fit presented in that work, a strong $\mathrm{C}_{\text {II }} \lambda 6580$ absorption was predicted, but the mismatch between the prediction and observations was attributed mainly to the poor $\mathrm{S} / \mathrm{N}$ in those spectra. The $\mathrm{S} / \mathrm{N}$ in the SNIFS spectrum is much higher, but the $C_{\text {II }} \lambda 6580$ is very weak. While NLTE effects may be in play (Thomas et al. 2007), we considered further alternative explanations for the $4130 \AA$ feature. An interesting possibility is CrII absorption-the inclusion of this ion also helps produce the strong emission at $4600 \AA$, and contributes to the absorption at $3280 \AA$, along with other iron-peak elements. $\mathrm{Cr}$ II is not without precedent in SN Ia spectra, though its appearance in more normal events seems limited to postmaximum epochs (Branch et al. 2008). However, detailed reconstruction of the 4000-4130 $\AA$ region remains difficult - in particular, Ni II is invoked to explain some of the UV line blanketing, but produces an extra notch at $3950 \AA$.

\subsection{Maximum-light Behavior, Colors and Extinction}

The rest-frame Bessell BVRIJ light curve of SN 2007if is given in Table 2 and in Figure 6. The color evolution is shown in Figure 7. An accurate estimate of the luminosity and ${ }^{56} \mathrm{Ni}$ mass for SN 2007if requires some care, due in part to the sparse multi-band light curve coverage around maximum light. In this section, we explore two different methods of interpolating the light curves to maximum, derive the observed color of the $\mathrm{SN}$, and comment briefly on reddening by dust in the host galaxy.

Fitting the multi-band light curve of SN 2007if with existing $\mathrm{SN}$ Ia templates trained on normal $\mathrm{SNe}$ Ia is of course problematic. As an example, the SALT2 model (Guy et al. 2007) fit to all four bands is poor $\left(\chi_{v}^{2}=930.3 / 122=7.6\right)$, above the threshold used by the SNLS to photometrically screen events

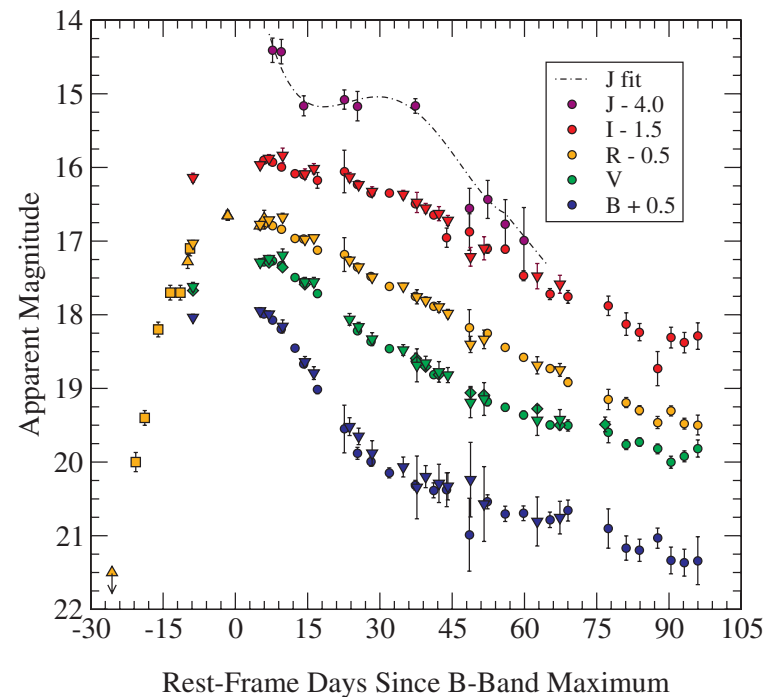

Figure 6. Rest-frame Bessell BVRIJ light curve for $\mathrm{SN}$ 2007if from ANDICAM+SNIFS+QUEST. Upright triangles: QUEST $R G 610, K$-corrected to rest-frame Bessell $R$. Diamonds: SNIFS $V$ from photometric imaging channel, $K$-corrected to rest-frame Bessell $V$. Inverted triangles: Rest-frame Bessell $B V R I$ magnitudes synthesized from SNIFS flux-calibrated spectroscopy. Circles: ANDICAM $B V R I J, K$-corrected to the respective rest-frame Bessell filters. Squares: ROTSE-III points from Akerlof et al. (2007). A fourth-order polynomial fit to the $J$-band data is also shown.

(A color version of this figure is available in the online journal.)

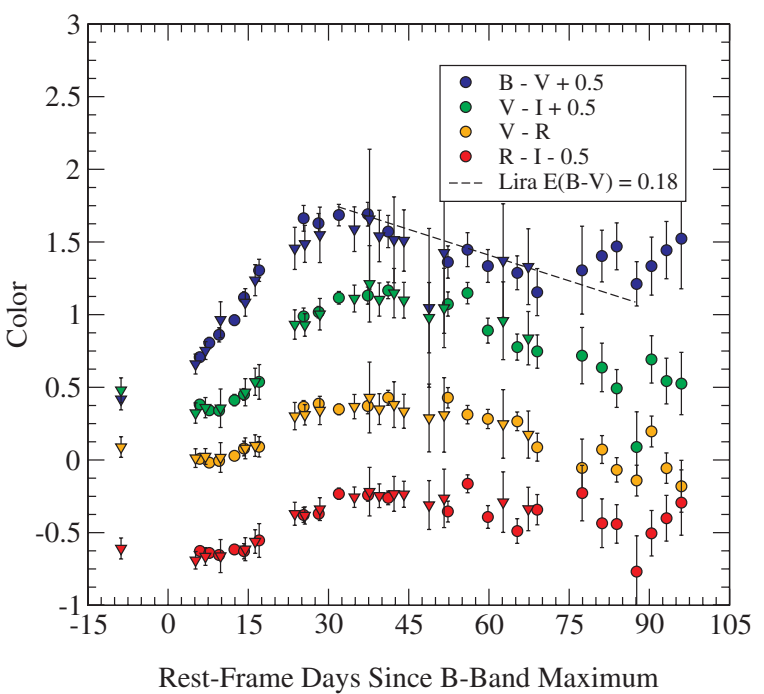

Figure 7. Rest-frame Bessell color evolution for SN 2007if from ANDICAM (circles) and SNIFS spectrophotometry (inverted triangles). A fit to the Lira relation with $E(B-V)=0.18$ is shown.

(A color version of this figure is available in the online journal.)

as "probable" SNe Ia (Sullivan et al. 2006). The event would therefore have been deprioritized for spectroscopic follow-up by SNLS and might not have been studied further. The fit is especially poor in $R$ and $I$, which lack the distinct second maximum typical of normal SNe Ia; such behavior also was noted in SN 2006gz by Hicken et al. (2007) and in SN 2009dc by Yamanaka et al. (2009).

We can nevertheless attempt to use SALT2 simply as a method for interpolating the shape of the light curve around maximum light, taking the $\chi^{2}$ and fitted parameters $x_{1}$ and $c$ provisionally. To minimize the impact of details of the SALT2 spectral model on the outcome, we use SALT2 in the rest frame, include SALT2 
light curve model errors in the fitting, and we fit $B V R$ bands only, excluding the clearly discrepant $I$. This procedure gives a more reasonable $\chi^{2} / v=144.8 / 93=1.6$, with a date of $B$-band maximum around MJD 54348.4 (2007 September 6), a stretch of 1.15 ( $x 1=1.83$, consistent with SN 2003fg) and a very red color $(c=0.24)$. The best-fit magnitudes at $B$-band maximum are $m_{B}=17.34 \pm 0.04, m_{V}=17.18 \pm 0.05$, $m_{R}=17.14 \pm 0.06$ (statistical $+K$-correction errors added in quadrature), giving $B-V=0.16$. The value of $m_{R}$ so derived is also consistent with the observed unfiltered magnitude at maximum (calibrated to USNO-B $1.0 R$; Monet et al. 2003) observed by ROTSE-IIIb (Akerlof et al. 2007) and the QUESTII $R G 610$ point at that date.

As a cross-check, we fit a cubic polynomial to the restframe $B V R$ points before MJD 54374.0 (three weeks after the SALT2 date of $B$-band maximum). This gives maximum magnitudes $m_{B}=17.33 \pm 0.03, m_{V}=17.25 \pm 0.03$, and $m_{R}=$ $17.19 \pm 0.02$, with the date of $V$-band maximum consistent with the SALT2 fits to within \pm 2 days. The uncertainties on the date of maximum are larger with this approach, since each band can vary independently; however, the results are roughly consistent with SALT2. We take the results of the SALT2 fit as our fiducial values in further discussion -in particular the phase of maximum light at MJD 54348.4, in which case the SMARTS observations begin at day +5 after maximum light.

Both of the above extrapolations imply that SN 2007if is redder than the typical SN Ia at maximum light. To assess the impact of dust extinction, we use the Lira relation (Phillips et al. 1999; Folatelli et al. 2010) and the equivalent width of Na I D absorption (Turatto et al. 2002) ("TBC relation"). Two possible slopes for the TBC relation are given, one shallow $(E(B-V) \sim$ $0.5 \times \mathrm{EW}(\mathrm{Na}$ I D $))$ and one steep $(E(B-V) \sim 0.15 \times$ $\mathrm{EW}(\mathrm{Na}$ I D)).

The $B-V$ color evolution of SN 2007if (see Figure 7) has a slope compatible with the Lira relation (Phillips et al. 1999) with a fitted color excess of $E(B-V)_{\text {host }}=0.18 \pm 0.04$ (stat) \pm 0.06 (sys). Assuming the Lira relation holds (which for this unusual SN is by no means clear), the intrinsic color of the $\mathrm{SN}$ is $B-V=-0.02$.

A noise-weighted co-add of the SNIFS spectra reveals no Na I $\mathrm{D}$ absorption at the redshift of the host. To derive an upper limit on EW(Na I D) while allowing for uncertainty in the redshift, we construct the probability surface over equivalent width and redshift for a joint fit of the Na I D line profile within the appropriate wavelength range for all observed spectra of SN 2007if. In the fit, the local continuum is estimated separately for each spectrum as a cubic polynomial, and the absorption is fully modeled using separate wavelengths for both $\mathrm{Na}$ I $\mathrm{D}$ lines using the SNIFS instrumental resolution of $6 \AA$. The resulting probability density was then marginalized over the redshift error to obtain the final estimate. For the Milky Way dust absorption, we derive $\mathrm{EW}(\mathrm{Na}$ I D $)=0.51_{-0.05}^{+0.04} \AA$, which under the "shallow" TBC relation corresponds to $E(B-V)=0.072$, in good agreement with the reddening from Schlegel et al. (1998). No absorption is detected from the host, and we derive an upper limit EW(Na I D) $<0.14 \AA$ (95\% CL), which corresponds to $E(B-V)<0.032$ even under the "steep" TBC relation.

A similar discrepancy between the Lira relation and $\mathrm{Na}$ I D absorption was noted by Yamanaka et al. (2009) for SN 2009dc; their work ended up adopting the estimate from EW(Na I D). Under the same assumption for SN 2007if, host reddening is therefore unlikely to account for a significant share of $\mathrm{SN}$ 2007if's deviation from the colors of normal SNe Ia. The color difference is probably real and intrinsic. If the entire color difference were due to reddening with $R_{V} \sim 3.1$, this would make SN 2007if nearly a full magnitude brighter at maximum than SN 2003fg. However, given the disagreement between the two host reddening estimates, we revisit the potential impact of uncontrolled host reddening on our results in Section 5.6. The primary analysis to follow in our paper assumes zero host galaxy reddening.

Adopting the magnitudes at maximum light from the SALT2 fits to the rest-frame light curve and a CMB-frame redshift of $z_{\mathrm{CMB}}=0.0731 \pm .0013$ (including a $300 \mathrm{~km} \mathrm{~s}^{-1}$ peculiar velocity) gives a Hubble-flow distance modulus of $\mu=37.57 \pm$ 0.03 for the concordance cosmology $\left(h=0.71, \Omega_{M}=\right.$ $0.27, \Omega_{\Lambda}=0.73$; see Wright 2006) implies absolute magnitudes $M_{B}=-20.23, M_{V}=-20.39$ assuming zero reddening from the host galaxy. This makes SN 2007if the brightest superChandrasekhar-mass SN Ia candidate yet discovered. The SN is at least some $1.3 \mathrm{mag}$ brighter in $V$ than a "normal" $x_{1}=0$ SN Ia of the same color, and too bright for its SALT2 value of $x_{1}$ by about $1.1 \mathrm{mag}$ (taking $M_{V}\left(x_{1}=0, c=0\right)=-19.07$, $\alpha=0.13, \beta=1.77$, after Guy et al. 2007).

The temporal sampling and $\mathrm{S} / \mathrm{N}$ in ANDICAM $J$ are much lower than in the optical bands, due to the limited time available in our SMARTS observing program. However, the light curve suggests a second maximum starting 15-20 days after $B$-band maximum light, lasting until about 40 days after $B$-band maximum light and then dropping dramatically. The height of the first maximum is not clear from the data; the second maximum probably occurs 28-34 days after maximum light, at a height of $J \sim 19$. Figure 6 shows a fit of a fourth-order polynomial to the data within the range of phase coverage, showing a possible position for the second maximum.

The estimate of $J$-band flux at maximum light is similarly problematic. If the $J$-band light curve near peak is similar to the behavior, e.g., of $R$-band, extrapolated backward nearly linearly without a pronounced maximum, then maximum light might be only as bright as the brightest observed point, or $J_{\max }=18.4$. If the first maximum is more pronounced, as in the high- ${ }^{56} \mathrm{Ni}$ light curves of Kasen (2006), then it could be as bright as $J_{\max }=17.8$. We take these estimates as representative lower and upper limits.

Given these estimates of the flux in different bands at $B$-band maximum light, and a spectrum similar to the spectrum at +5 days, we estimate a maximum bolometric luminosity of $(3.22 \pm 0.15) \times 10^{43} \mathrm{erg} \mathrm{s}^{-1}$.

\subsection{Light Curve Comparison to SN $2003 \mathrm{fg}$}

Figure 8 shows a direct comparison between the light curves of SN 2007if and SN 2003fg. Since a spectral time series which might yield accurate $K$-corrections for SN 2003fg is not available, we have instead $K$-corrected our SN 2007if light curve to the observer-frame gri bandpasses of SN 2003fg at $z=0.244$.

We can see from this comparison that SN 2007if and SN $2003 \mathrm{fg}$ have very similar decay behavior. SN $2003 \mathrm{fg}$ is certainly fainter than SN 2007if by about $0.3 \mathrm{mag}$. The relative color and light-curve phase between the two $\mathrm{SNe}$ is uncertain due to the lack of maximum-light coverage in $g$ band for SN 2003fg. SN 2003 fg is probably bluer than SN 2007if, but not by more than about $0.1 \mathrm{mag}$, or $B-V=0.06$; this is difficult to reconcile with the reported $B-V=-0.15$ (Howell et al. 2006; Hicken et al. 2007). We shall return to the interpretation of possible color differences between the two SNe below; it is unclear that the excess should be interpreted in terms of dust extinction. 


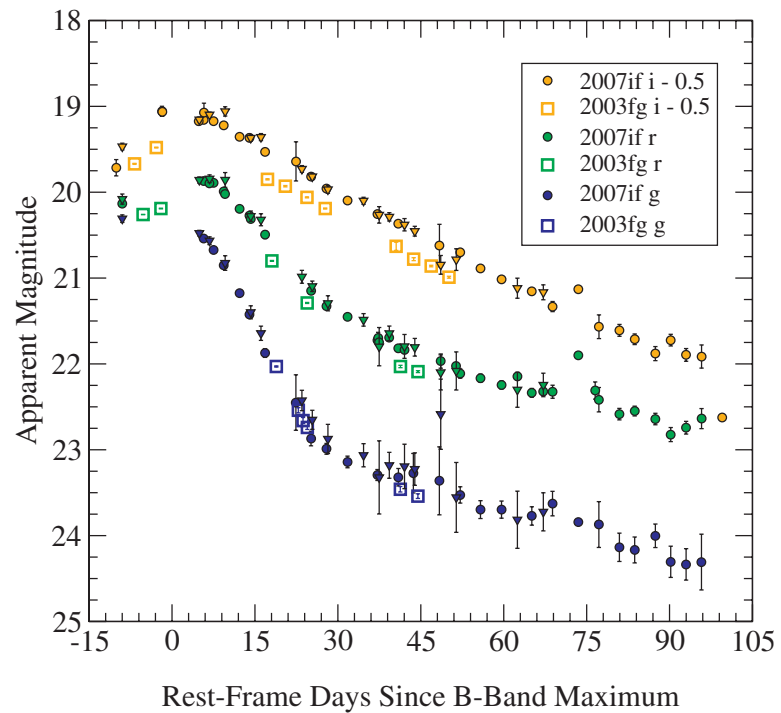

Figure 8. Direct comparison of SN 2007if multi-band light curves with SN 2003fg in the latter's observer frame. Solid circles: ANDICAM+QUEST+ SNIFS $B V R$ for SN 2007if, $K$-corrected to SNLS gri at $z=0.244$. Open squares: SNLS gri observer-frame $(z=0.244)$ light curves for SN $2003 \mathrm{fg}$ (see Howell et al. 2006).

(A color version of this figure is available in the online journal.)

\section{SYNTHESIZED NICKEL MASS IN SN 2007IF}

The maximum magnitude $R=17.2$ observed in the search and follow-up images suggest a highly overluminous $\left(M_{V} \sim\right.$ -20) explosion for SN 2007if, similar to SNe 2003fg and $2009 \mathrm{dc}$. This in turn suggests a very large mass of ${ }^{56} \mathrm{Ni}-$ could it be in excess of $M_{\mathrm{Ch}}$ ? In this section, we calculate a bolometric light curve of the SN, and from an estimate of the bolometric luminosity at maximum light, derive the ${ }^{56} \mathrm{Ni}$ mass using Arnett's rule (Arnett 1982).

\subsection{Bolometric Light Curve}

Bolometric light curves of SNe Ia are useful for estimating the ${ }^{56} \mathrm{Ni}$ mass (Arnett 1982), from the bolometric luminosity at peak, and the total ejected mass, from an estimate of the energy deposition in the ejecta from decay of radioactive ${ }^{56} \mathrm{Ni}$ and ${ }^{56} \mathrm{Co}$ (Stritzinger et al. 2006). Maeda \& Iwamoto (2009) characterize each SN by the decay time of the bolometric light curve.

To produce a bolometric light curve for a given SN Ia, Stritzinger et al. (2006) use a parameterized model to interpolate the multi-band light curve in time, which is then numerically integrated in wavelength to find the total flux over bands as a function of time. We choose to take a slightly different approach using the observed spectra of SN 2007if, similar to that used by Howell et al. (2009). For each quasi-simultaneous set of ANDICAM BVRI observations, we deredden and deredshift the SNIFS spectrum nearest in time, then multiply it by a smooth function (in this case a cubic polynomial) fitted so that the synthetic photometry from the resulting spectrum matches, in a least-squares sense, the corresponding ANDICAM imaging photometry in each band. We then calculate the bolometric flux as the integral of the SNIFS spectrum over all rest-frame wavelengths from $3100-9000 \AA$. Occasionally $(<10 \%$ of the time) an ANDICAM observation in one band is missing due, e.g., to $\mathrm{S} / \mathrm{N}$ considerations from intermittent clouds or bright moon-

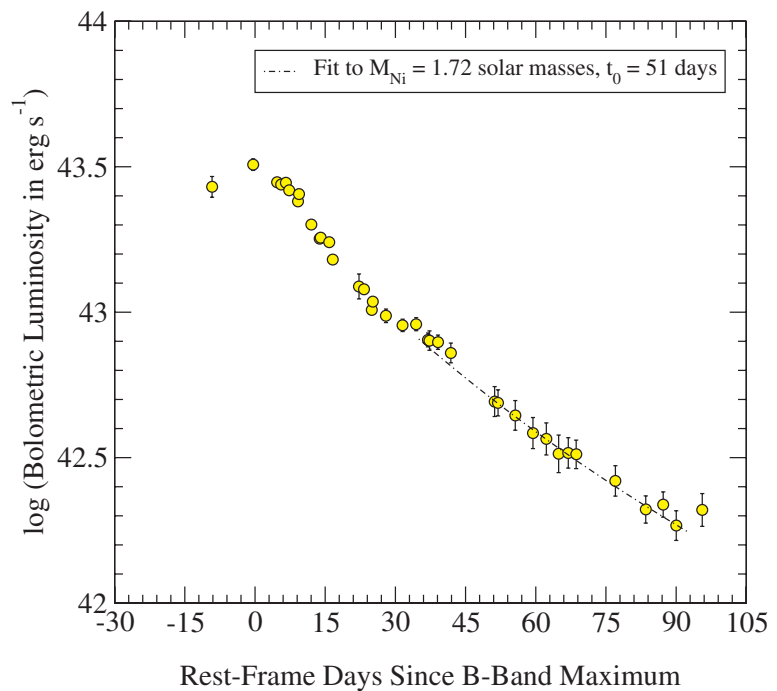

Figure 9. Rest-frame bolometric light curve of SN 2007if, synthesized from SMARTS BVRIJ data, SNIFS spectroscopy, and NIR spectra of SN 1999ee taken with ISAAC on the VLT at Cerro Paranal (Hamuy et al. 2002). A fit of the radioactive energy deposition curve to the data taken more than 60 days after explosion is shown.

(A color version of this figure is available in the online journal.)

light; since the phase coverage is overall still exemplary, such gaps are covered via linear interpolation between adjacent light curve points, and a statistical error bar assigned to the interpolated value based on the $\mathrm{S} / \mathrm{N}$ of the adjacent points. This never occurs in more than one of the bands at a time. As a cross-check on the effects of the evolution of spectral features on the bolometric flux thus reconstructed, we evaluated each bolometric light curve point using the two spectra bracketing it in time, and took the difference between the measurements as an estimate of the systematic error. This difference was found always to be less, and usually much less, than the statistical error on each light curve point.

This handles only the optical section of the light curve, however. Given the discussion in Section 5.2 below, we can expect much of the flux to be reprocessed into the NIR. To account for this, we repeat the above procedure for the NIR, by normalizing the synthetic $J$ magnitudes over the spectra of SN 1999ee to match the observed $J$-band photometry. We expect systematic errors resulting from this approximation to be small, since SN 1999ee is itself an overluminous LVG SN Ia. We then integrate the flux from 9700 to $24800 \AA$. To compensate for the poor phase coverage and $\mathrm{S} / \mathrm{N}$ in ANDICAM $J$, we normalize to the fourth-order polynomial fit in Figure 6. We estimate, or place limits on, the $J$-band contribution outside the bounds of phase coverage with a piecewise linear extrapolation based on the $J$-band light curves from Kasen (2006). Most of the high- ${ }^{56} \mathrm{Ni}$ models show a decline of $0.08 \mathrm{mag} \mathrm{day}^{-1}$ after the second peak, which we adopt for rest-frame day +55 to day +70 . The subsequent behavior is uncertain and we allow for the possibility of a decline anywhere in the range 0.02-0.06 mag day ${ }^{-1}$. These limits and uncertainties are propagated into the bolometric light curve error bars. We find that the NIR correction to the bolometric luminosity ranges from $30 \%$ of the optical luminosity (near the second maximum) to $10 \%-15 \%$ after day +60 . The NIR correction is less than $5 \%$ around $B$-band maximum light, so that the expected systematic error on the ${ }^{56} \mathrm{Ni}$ mass is small.

The resulting bolometric light curve is shown in Figure 9. 


\subsection{Estimate of the ${ }^{56} \mathrm{Ni}$ Mass from Arnett's Rule}

To estimate the ${ }^{56} \mathrm{Ni}$ mass, we calculate the rate of radioactive energy deposition in the ejecta,

$$
\begin{aligned}
& L_{\mathrm{rad}}=N_{{ }^{56} \mathrm{Ni}} \lambda_{56}{ }_{\mathrm{Ni}} Q_{56}{ }_{6 \mathrm{Ni}, \gamma} \exp \left(-\lambda_{56} \mathrm{Ni} t\right) \\
& +N_{56}{ }_{\mathrm{Ni}} \frac{\lambda_{56} \mathrm{Co} \lambda_{56} \mathrm{Ni}}{\lambda_{56}{ }_{\mathrm{Ni}}-\lambda_{56} \mathrm{Co}}\left(Q^{56} \mathrm{Co}, e^{+}+Q_{56 \mathrm{Co}, \gamma}\right) \\
& \times\left(\exp \left(-\lambda_{{ }_{56} \mathrm{Co}_{\mathrm{o}}} t\right)-\exp \left(-\lambda_{56} \mathrm{Ni}_{\mathrm{i}} t\right)\right),
\end{aligned}
$$

where $t$ is the time since explosion, $N_{56 \mathrm{Ni}}=M_{56} \mathrm{Ni} /(56 \mathrm{AMU})$ is the number of ${ }^{56} \mathrm{Ni}$ atoms produced in the explosion, $\lambda_{56} \mathrm{Ni}$ and $\lambda_{56} \mathrm{Co}$ are the decay constants for ${ }^{56} \mathrm{Ni}$ and ${ }^{56} \mathrm{Co}$ (lifetimes 8.8 days and 111.1 days) respectively, and $Q_{56 \mathrm{Ni}, \gamma}, Q_{56} \mathrm{Co}, \gamma$, and $Q_{56}{\mathrm{Co}, e^{+}}$are the energies released in the different stages of the decay chain (Nadyozhin 1994). We adopt an efficiency factor $\alpha$ of order unity such that $L_{\mathrm{bol}}=\alpha L_{\mathrm{rad}}$ (Höflich \& Khohklov 1996; Jeffery et al. 2006). We use a fiducial value of $\alpha=1.2$ (Jeffery et al. 2006; Howell et al. 2006, 2009), within the range $0.8<\alpha<1.6$ representative of various models (Jeffery et al. 2006). Given the quality of our data, the uncertainty on $\alpha$ is the limiting systematic error in our analysis.

Since ROTSE-IIIb detected the SN at unfiltered magnitude $19.5 \pm 0.1$ as early as August 16.3 UT (Akerlof et al. 2007; F. Yuan et al. 2010, in preparation), the observer-frame rise time must be at least 20 days. Palomar-QUEST detects no object at the SN location on August 9.0, however, placing an upper limit of $R G 610>21.5$ at $95 \%$ confidence; thus the observer-frame rise time cannot be any longer than 26 days. A parabolic (Riess et al. 1999; Aldering et al. 2000) fit to the first four points on the ROTSE light curve (Akerlof et al. 2007) suggests an explosion date of 2007 August 10.7 (MJD 54322.6); this gives a rest-frame rise time of $24.2 \pm 0.4$ days, which we adopt for our analysis.

Using these numbers we derive a ${ }^{56} \mathrm{Ni}$ mass of $M_{56} \mathrm{Ni}=$ $(1.72 \pm 0.09) \times(\alpha / 1.2) M_{\odot}$, somewhat larger than SN $2003 \mathrm{fg}$ and SN 2009dc, and already in excess of the Chandrasekhar mass for $\alpha=1.2$. This estimate assumes no extinction due to dust in the host galaxy. The Chandrasekhar-mass DET1 model of Khokhlov et al. (1993), in contrast, makes only $0.92 M_{\odot}$ of ${ }^{56} \mathrm{Ni}$, approximately the theoretical upper limit for Chandrasekharmass detonations. We will return to the ${ }^{56} \mathrm{Ni}$ mass estimate below in the context of more detailed models of the SN 2007if progenitor.

\section{CONSTRAINTS ON THE SN 2007IF TOTAL MASS AND DENSITY STRUCTURE}

There have been several attempts to explain how a Chandrasekhar-mass white dwarf in a SD scenario could give rise to an unusually luminous explosion, usually relying on departures from spherical symmetry. Hillebrandt et al. (2007) simulated aspherical three-dimensional SN Ia explosions, which at their brightest achieved $M_{\mathrm{bol}}=-19.9$ at maximum, but at the cost of some fine-tuning and requiring an almost complete conversion of the star to ${ }^{56} \mathrm{Ni}$. They also failed to reproduce the low velocities found in SN 2003fg-like SNe. Kasen et al. (2004) considered initially spherical explosions which lost spherical symmetry through the interaction of the SN ejecta with a non-degenerate companion star, forming a conical hole through which deeper, hotter ejecta became visible along certain lines of sight; such a model produces a SN with a 1991T-like spectrum, bluer colors and only a modest $(\sim 0.3 \mathrm{mag})$ luminosity enhancement relative to normal SNe Ia. Neither of these models are consistent with our observations of SN 2007if.
Tanaka et al. (2009) present polarimetry observations for SN $2009 \mathrm{dc}$ which show no significant departures from spherical symmetry. This would seem to imply that, whether or not the progenitor star is aspherical, a highly aspherical explosion is not necessary to attain a very high luminosity. However, it is reasonable to ask whether observations can provide further constraints on the mass of the progenitors in such systems apart from measuring the SN's luminosity and velocity near peak, within the context of an explosion with no large-angular-scale anisotropies. One way of approaching this question is via the late-time bolometric light curve (Jeffery 1999; Stritzinger et al. 2006), which requires an accurate estimate of the kinetic energy of the explosion and the corresponding velocity scale for the ejecta density profile.

Our measurements (see Figure 4) show an unusually low Si II $\lambda 6355$ velocity in the ejecta, as has been seen in other super-Chandrasekhar SN Ia candidates. We also see a fairly flat velocity evolution, consistent with a plateau in the Si II velocity $\left(\delta v \leqslant 500 \mathrm{~km} \mathrm{~s}^{-1}\right.$; Quimby et al. 2007) starting as early as day -9 and extending at least as late as day +7 . These low velocities have previously been attributed to large binding energies for these events (Howell et al. 2006). In the following section we explore an alternative- that the low velocity might result from deceleration of the outer layers of ejecta by a massive envelope surrounding the SN progenitor, producing an overdense shell in the ejecta. We present a simple parameterized treatment of the shell structure which allows us to constrain the true underlying kinetic energy of the explosion, the mass of the shell and the envelope, and the total ejected mass.

\subsection{Shell Structure in SN 2007if}

Quimby et al. (2007) studied the influence of shell structure in SN Ia ejecta for a normal SN Ia event, SN 2005hj. They pointed out that an overdense spherical shell in the ejecta expanding at a given velocity slows the recession of the photosphere, producing a plateau at that velocity in the time evolution of the widths of absorption features, e.g., Si II. The shell would also be characterized by a duration and a velocity-space width from the spectra. Photometrically, SNe Ia with an overdense shell in the outer layers should appear redder and brighter than SNe Ia with no shell. Existing models of shell-structure $\mathrm{SNe}$ Ia have been calculated only for Chandrasekhar-mass explosions, but one should expect analogous physical effects in super-Chandrasekhar-mass explosions. Such models provide an alternate interpretation for the low Si II velocities seen in SN 2003fg and SN 2007if, although a large progenitor binding energy may also contribute.

Shell structure occurs naturally in DD merger scenarios, in which the tidal disruption of one or both white dwarfs creates a common envelope of carbon and oxygen around the central merger product. Collision of the ejecta with the envelope then accelerates the envelope to higher velocities and creates a strong reverse shock which creates a shell in the outermost SN ejecta. Höflich \& Khohklov (1996) simulate several instances of common envelopes in their DET2ENVN "tamped detonation" (TD) models, which place two merging white dwarfs of total mass 1.2 solar masses in an envelope of mass $0.1 \times N M_{\odot}$. DET2ENV4 and DET2ENV6 produce $B-V \sim 0.2$ near maximum light, significantly redder than SN 2006gz but consistent with 2007if. The DET2ENV6 model, with a total system mass of $1.8 M_{\odot}$, also has $\Delta m_{15}(V)=$ $0.63_{-0.09}^{+0.15}$, the slowest of any model in that work, compared with $\Delta m_{15}(V)=0.50 \pm 0.07$ for SN 2007if. (The number 
for SN2007if is interpolated from the observations using the SALT2 fit; the dominant source of error for both the models and the observations comes from the uncertain phase of maximum light).

The SD pulsating delayed detonation (PDD) models of Höflich \& Khohklov (1996) also have shell structure in the ejecta. Here an initial deflagration phase pre-expands the white dwarf; the outermost layers begin to recollapse and are then entrained into a shell by the underlying ejecta from a subsequent detonation. These models do exhibit an intrinsically red $B-V$ color at maximum, though with a wide spread (0.05-0.60). However, they have unusually short rise times (13-15 days) and slower declines than observed for SN 2007if. Most of the Höflich \& Khohklov (1996) models have rise times which are too short-around 15 days in $V$, compared to the observed typical value of 19 days (Conley et al. 2006). This aspect of the models could no doubt be improved for a more accurate match to observations. Nevertheless, it is striking that the shell-structure explosion models reach the extremes of the rise time distribution with fairly small dispersion, with most PDD models at 13 days and the TD models as large as 22 days. The one PDD model that achieves a sufficiently long rise time and slow decline, PDD535, is much fainter even than normal SNe Ia $\left(M_{V}=-17.77\right)$ and has an extremely red color of $B-V=0.60$. This model produces about $20 \%$ of the kinetic energy of the DET2ENVN models.

A separate question arises regarding the widespread use of the differentially rotating models of Yoon \& Langer (2005) in treatments of super-Chandrasekhar-mass SNe Ia (Howell et al. 2006; Jeffery et al. 2006; Maeda \& Iwamoto 2009), including this paper (see below). Piro (2008) found that inclusion of the baroclinic instability and of magnetohydrodynamic processes in white dwarf interiors inhibits the Kelvin-Helmholtz instabilities expected in differentially rotating white dwarfs, limiting such white dwarfs to rigid rotation and apparently making SD superChandrasekhar-mass white dwarfs untenable. Even discounting such effects, however, supermassive white dwarfs accreting from a non-degenerate companion are not expected to evolve to masses above $1.7 M_{\odot}$ (Chen \& Li 2009); the progenitor of SN 2007if was almost certainly more massive than this.

The interpretation of a velocity plateau as the sign of an interaction with an envelope containing a few tenths of a solar mass of material also poses problems for models involving the explosion of SD supermassive white dwarfs. To the extent that such an envelope is necessary to explain the kinematics of 2003 fg-like SNe Ia, it must be composed of carbon and oxygen; a hydrogen/helium envelope of comparable mass would most likely produce strong emission lines such as those seen in the "SNe IIa" 2002ic (Hamuy et al. 2003; Wood-Vasey et al. 2004) and 2005gj (Aldering et al. 2006; Prieto et al. 2007).

The above considerations disfavor existing SD explosion models, although it is conceivable that some PDD model could be tuned to fit our observations. The TD models provide a better fit to the observations. For further analysis below, we therefore discount models of PDDs for the time being, and concentrate instead on TDs representing DD mergers, using DET2ENVN as our main point of reference.

\subsection{Near-IR Light Curves and Mixing of the Ejecta}

Our estimate of the total ejected mass will follow Jeffery (1999) and Stritzinger et al. (2006), relying on the estimated transparency of the ejecta to gamma rays from ${ }^{56} \mathrm{Co}$ decay. The model requires an assumption about the distribution of ${ }^{56} \mathrm{Ni}$ in the ejecta. Although this incurs some uncertainty, we argue that the ejecta of SN 2007if are fairly well mixed based on the near-infrared (NIR) light curves.

Kasen (2006) presents radiative transfer Monte Carlo calculations which illustrate the relevance of mixing in SN Ia ejecta to the appearance of an inflection point or second maximum in their NIR light curves. The recombination of iron provides an efficient means of redistributing light from UV and blue bands to the NIR, and recombination happens at a more or less sharp front in SN Ia ejecta as they expand and cool. Thus in SNe with compact cores of iron-peak elements, a wave of recombination of Fe III to Fe II powers the first NIR light curve peak, while the recombination of Fe II to Fe I powers the well-known second peak. SNe in which the ejecta are well-mixed have less pronounced second maxima in the near infrared than those in which the ejecta are stratified. SNe which produce less ${ }^{56} \mathrm{Ni}$ also show less pronounced second maxima, and these maxima occur earlier in phase and are more closely spaced with respect to the first maximum, since the temperature of the ejecta is lower and recombination of iron-peak elements occurs earlier.

The I-band light curve of 2007if shows only a very slight inflection which is difficult to measure. The $J$-band light curve shows stronger, but still quantitatively weak, evidence for a second maximum. In the context of this model, the observed $I$ - and $J$-band light curves of SN 2007if suggest either a low ${ }^{56} \mathrm{Ni}$ mass, ejecta which are highly mixed, or both. Assuming stratified ejecta, the shape, timing and duration of the $J$-band second peak is broadly consistent with a large amount of ${ }^{56} \mathrm{Ni}$ ( $0.6 M_{\odot}$ or more). Given the luminosity of the event, a plausible interpretation is that the ejecta contain a large amount of ${ }^{56} \mathrm{Ni}$, much of which lies near the surface-either because it has been mixed to higher velocities in the explosion, or because the ejecta are composed mostly of ${ }^{56} \mathrm{Ni}$ and little else. This interpretation is also supported by the faster decline in $B$ band relative to SN 2003fg, suggesting that the $B$-band flux is indeed being efficiently reprocessed to NIR wavelengths (Kasen \& Woosley 2007); a smaller amount of ${ }^{56} \mathrm{Ni}$ might manifest as a sharper decline in all bands (i.e., the bolometric light curve) rather than $B$ band only. It is also consistent with the appearance of Fe III lines near maximum, as mentioned in Section 2.1.

Khokhlov et al. (1993) point out that in SNe with shell structure, the shock interface between the shell and the highvelocity ejecta is Rayleigh-Taylor unstable. This could provide a mechanism for mixing of iron-peak elements into the shell in SN 2007if. If so, we might expect the associated features-a weak NIR second maximum and a relatively sharp $B$-band decline rate-to be a generic feature even of shell-structure $\mathrm{SNe}$ with less massive progenitors. We might therefore also expect the usual width-luminosity relationship to break down for shell-structure SNe Ia (Quimby et al. 2007), producing SNe which are systematically brighter for their ( $B$-band) decline rate than SNe Ia with near-exponential density structures (Kasen $\&$ Woosley 2007). Whether the bolometric light curves still followed a width-luminosity relationship would depend in part on the efficiency of the optical-NIR reprocessing.

\subsection{Mass Estimates and Photospheric Evolution for SN Ia Models with Exponential Density Profiles}

In the optically thin limit, and when the gamma rays come from a single radioactive species, Equation (2) is modified by multiplying the $Q_{\mathrm{Co}, \gamma}$ term by a factor $1-\exp \left(-\tau_{\gamma}\right)$, where $\tau_{\gamma}$ is the optical depth to scattering of gamma rays from the decay of ${ }^{56} \mathrm{Co}$. Such approximations are useful in describing the state of 
the ejecta by day +60 after explosion (Jeffery 1999; Stritzinger et al. 2006), by which time less than $0.5 \%$ of the initial ${ }^{56} \mathrm{Ni}$ should remain.

The optical depth $\tau_{\gamma}$ should scale as $(t)^{-2}$ for SN ejecta in homologous expansion. The evolution of the ejecta density in a SN Ia can be well described by

$$
\rho(v, t)=\rho_{\mathrm{c}, 0}\left(t_{0} / t\right)^{3} \exp \left(-v / v_{e}\right),
$$

where $\rho_{\mathrm{c}, 0}$ is the central density at fiducial time $t_{0}$ and $v_{e}$ is the scaling velocity. By taking $t_{0}$ to be the time at which $\tau_{\gamma}=1$, i.e., the transition to the optically thin regime (Jeffery 1999) and integrating outward from the center of the $\mathrm{SN}$, we obtain $\rho_{\mathrm{c}, 0}=\left(\kappa_{\gamma} q v_{e} t_{0}\right)^{-1}$, where $\kappa_{\gamma}$ is the opacity for gamma-ray absorption, and $q$ is a form factor describing how the ${ }^{56} \mathrm{Ni}$ is distributed throughout the ejecta. The total mass is

$$
M_{\text {ejected }}=8 \pi \rho_{\mathrm{c}, 0}\left(v_{e} t_{0}\right)^{3},
$$

and the kinetic energy is

$$
E_{K}=6 M_{\text {ejected }} v_{e}^{2} .
$$

We can then use these relations to estimate the total mass as

$$
M_{\text {ejected }}=\frac{8 \pi}{\kappa_{\gamma} q}\left(v_{e} t_{0}\right)^{2} .
$$

In their analysis of a sample of normal SN Ia bolometric light curves, Stritzinger et al. (2006) adopt $q=0.33 \pm$ 0.09 (appropriate for ejecta in which ${ }^{56} \mathrm{Ni}$ is mixed evenly throughout), $\kappa_{\gamma}=0.025 \pm 0.03 \mathrm{~g} \mathrm{~cm}^{-2}$, and $v_{e}=3000 \mathrm{~km} \mathrm{~s}^{-1}$. They emphasize that the procedure tends to produce low ejected masses $\left(<1.0 M_{\odot}\right)$ for many $\mathrm{SNe}$ Ia, and comment on the accuracy of the input parameters. The values of $\kappa_{\gamma}$ and $q$ are not expected to vary much, and the largest single uncertainty is expected to arise from the value of $v_{e}$ used, which may have uncertainties on the order of $20 \%$ and is squared in the above expression.

For ejecta following an exponential density distribution, the photospheric velocity $v_{\text {phot }}$ can also be calculated given $v_{e}$ by solving $\tau\left(v_{\text {phot }}\right)=2 / 3(\sim 50 \%$ escape probability $)$. This leads to

$$
v_{\mathrm{phot}}=v_{e} \ln \left(\frac{3 \kappa_{\mathrm{opt}} M_{\mathrm{WD}}}{16 \pi v_{e}^{2} t^{2}}\right),
$$

where $\kappa_{\mathrm{opt}}$ is now the opacity for optical photons. For a nearexponential density profile, we would expect Si II $\lambda 6355$ to track the photosphere (and hence $v_{e}$ ) for observations near maximum light; this is the assumption made by Maeda \& Iwamoto (2009) in their parameter study of super-Chandrasekhar-mass SNe Ia.

\subsection{Incorporating the Effects of a Shell}

The ejected mass estimate in Section 5.3 hinges on the assumption that the ejecta follow an exponential density distribution with velocity. This is no longer true for $\mathrm{SNe}$ with shell structure. However, the DET2ENVN models have an essentially undisturbed exponential density structure in the centrally concentrated ejecta underneath the shell. Although the presence of a shell modifies the velocity structure and colors of the SN considerably, it should have negligible effect on the bolometric light curve at sufficiently late times, since the opacity $k_{\gamma}$ is an order of magnitude smaller than $k_{\mathrm{opt}}$ in most realistic scenarios. Due to geometrical dilution, the shell becomes transparent to gamma rays by maximum light, after which the exponential models should apply. The shell mass should consist simply of the ejected mass above some velocity $v_{\text {sh }}$, which looks like

$$
\frac{M\left(v>v_{\mathrm{sh}}, t\right)}{M_{\mathrm{WD}}}=Q\left(3, \frac{v_{\mathrm{sh}}}{v_{e}}\right),
$$

where $Q(a, x)=\gamma(a, x) / \Gamma(a)$ is the incomplete gamma function. Similar gamma-function integrals can be calculated to give the fractions of the optical depth, momentum, and kinetic energy, for which the values of $a$ are 1, 4, and 5, respectively. For a typical model with $v_{e}=2750 \mathrm{~km} \mathrm{~s}^{-1}$, the material above $9000 \mathrm{~km} \mathrm{~s}^{-1}$ carries about $35 \%$ of the ejected mass and $75 \%$ of the kinetic energy, yet contributes less than 5\% to the gammaray optical depth as measured from the center of the explosion to infinity.

We therefore expect that the bolometric light curve will yield a reasonable mass estimate for SN 2007if, provided we choose $v_{e}$ appropriately. However, in the presence of a shell, the Si II velocity is not representative of the kinetic energy velocity $v_{e}$ during the velocity plateau, but instead represents the shell velocity. Our observations show no significant change in the $\mathrm{Si}$ II velocity over a 16 day period ( -9 days to +7 days), inconsistent with the photospheric evolution of a nearexponential density profile even for SNe Ia with very low kinetic energy. Although Si II $\lambda 6355$ is sometimes used at late times ( +40 days) to estimate $v_{e}$, the line is too heavily blended with other features in the SN 2007if post-plateau spectra to measure its velocity. We therefore cannot observe $v_{e}$ directly in our work here, and instead marginalize over it as a nuisance parameter.

For DD progenitor models with envelopes, we can derive the shell and envelope masses given $v_{e}$ via conservation of momentum and optical transparency arguments. Shortly after explosion, all $\mathrm{SN}$ ejecta above $v=v_{\mathrm{sh}}$ are coasting freely and the envelope with mass $M_{\text {env }}$ is stationary. At late times, the envelope has been accelerated to higher velocities, and the outermost SN ejecta are moving together in a shell of negligible thickness with velocity $v_{\text {sh }}$ (which will match our measured Si II velocities). In the DET2ENVN models, the specific momentum in the $\mathrm{C} / \mathrm{O}$ envelope is some $50 \%$ higher than in the shell. Conservation of momentum then gives

$$
\begin{aligned}
p_{\text {initial }} & =3 M_{\mathrm{WD}} v_{e} Q\left(4, \frac{v_{\mathrm{sh}}}{v_{e}}\right)=p_{\text {final }} \\
& =\left[M_{\mathrm{WD}} Q\left(3, \frac{v_{\mathrm{sh}}}{v_{e}}\right)+1.5 M_{\mathrm{env}}\right] v_{\mathrm{sh}} .
\end{aligned}
$$

Some algebra then gives

$$
M_{\mathrm{env}}=\frac{2}{3}\left[\frac{3 v_{e}}{v_{\mathrm{sh}}} Q\left(4, \frac{v_{\mathrm{sh}}}{v_{e}}\right)-Q\left(3, \frac{v_{\mathrm{sh}}}{v_{e}}\right)\right] M_{\mathrm{WD}} .
$$

The sum $M_{\mathrm{tot}}=M_{\mathrm{env}}+M_{\mathrm{WD}}$ should then equal the mass of the two white dwarfs in the original close binary system. The combined envelope-plus-ejecta shell should remain opaque for a time

$$
t_{\mathrm{sh}}=\frac{1}{v_{\mathrm{sh}}} \sqrt{\frac{\kappa_{\mathrm{opt}} M_{\mathrm{sh}}}{4 \pi}} .
$$

We can calculate $t_{\mathrm{sh}}$, assuming fractions $f_{\mathrm{IPE}}$ and $f_{\mathrm{IME}}$ of ironpeak $(\mathrm{Ni}, \mathrm{Co}, \mathrm{Fe})$ and intermediate-mass elements (including 
carbon and oxygen) respectively, by using the approximate lineopacity prescription of Mazzali et al. (2001),

$$
\kappa_{\mathrm{opt}}=0.5\left[0.25 f_{\mathrm{IPE}}+0.025 f_{\mathrm{IME}}\right]
$$

(see also Maeda \& Iwamoto 2009). Our use of this relation assumes the material in the shell has been mixed. The conserved momentum and the observed transparency of the shell provide indirect constraints on the underlying, unobserved $v_{e}$.

\subsection{Confidence Regions on the Progenitor Mass}

In our analysis, following Jeffery (1999), we adopt $\kappa_{\gamma}=$ $0.029 \mathrm{~g} \mathrm{~cm}^{-3}$ and $q=0.33$ (since an even distribution of ${ }^{56} \mathrm{Ni}$ is supported by our observed NIR light curves). The appropriate value for $v_{e}$ will depend on the kinetic energy, which in turn will depend on the mass and on the composition of the ejecta. To get a self-consistent mass estimate, we generate a suite of semianalytic progenitor models similar to those presented in Jeffery et al. (2006), but including an envelope with characteristics similar to those found in DET2ENVN. We parameterize our models by the white dwarf mass $M_{\mathrm{WD}}$ and the fractions $f_{i}$ of ${ }^{56} \mathrm{Ni}$, other iron-peak elements ("Fe"), intermediate-mass elements ("Si") and unburned carbon and oxygen ("C/O"), the radioactive energy deposition parameter $\alpha$, and the ratio $f_{\text {env }}$ of the $\mathrm{C} / \mathrm{O}$ envelope mass $M_{\text {env }}$ to the ejected mass $M_{\mathrm{WD}}$ which contributes to the original binding energy. The binding energy was calculated from the differentially rotating white dwarf models of Yoon \& Langer (2005), and the kinetic energy was calculated as the difference between the binding energy and the nuclear energy released.

After calculating $v_{e}$ for each model, we could then make predictions for the observed bolometric light curve to be compared directly with observations. In matching the bolometric luminosity at peak (Arnett's rule) jointly with the other observations, we sample values of $\alpha$ between 0.8 and 1.6 to cover a reasonable range of potential variation in different explosion scenarios. However, for our primary analysis we adopt a fiducial value of $\alpha=1.3$, characteristic of the TD models DET2ENVN which lie within \pm 0.05 of this value (Höflich \& Khohklov 1996). We calculate $v_{\mathrm{sh}}$ given $v_{e}$ and $M_{\mathrm{env}}$ by solving Equation (8) numerically, and we calculate $t_{\mathrm{sh}}$ from Equations (9) and (10) (using $f_{\mathrm{IPE}}=f_{\mathrm{Fe}}+f_{\mathrm{Ni}}$ and $f_{\mathrm{IME}}=f_{\mathrm{Si}}+f_{\mathrm{CO}}$ ). In constraining the models we require that $v_{\mathrm{sh}}$ match the observations (9000 \pm $500 \mathrm{~km} \mathrm{~s}^{-1}$ ) and that $t_{\mathrm{sh}}$ be longer than the observed lifetime of the plateau ( +7 days after $B$-band maximum, or +32 days after explosion). Although the spectral characteristics begin to change at day +10 after $B$-band maximum, including a weakening of Si II $\lambda 6355$ which signals the end of the plateau phase, we do not enforce a hard upper limit on the length of the plateau.

The value of $M_{\text {tot }}$ was sampled uniformly from 1.4 to $2.8 M_{\odot}$, with the highest allowed mass corresponding to the merger of two Chandrasekhar-mass white dwarfs. We chose $2 M_{\mathrm{Ch}}$ as the most conservative upper bound on the system mass, since observations, or the theory of common-envelope evolution thought to produce DD systems, do not yet provide secure constraints (see, for example, Dobbie et al. 2006, 2009). The values $f_{\mathrm{Fe}}, f_{\mathrm{Ni}}, f_{\mathrm{Si}}$, and $f_{\mathrm{CO}}$ (representing the composition of the bound progenitor mass) were sampled uniformly between 0 and 1 such that their sum was unity. The value of $f_{\mathrm{env}}=M_{\mathrm{env}} / M_{\mathrm{WD}}$ was varied independently between 0 and 0.5 . The central density of the merged progenitor was fixed at $3 \times 10^{9} \mathrm{~g} \mathrm{~cm}^{-3}$, higher than the DET2ENVN models but typical of central densities of white dwarfs assumed elsewhere (Howell et al. 2006; Maeda
\& Iwamoto 2009). For models with $f_{\text {env }}>0$, i.e., models with shells, we assume that all of the IME are mixed into the shell, to agree with our observations of very weak or absent $\mathrm{Si}$ II in post-plateau spectra.

To generate confidence regions, we evaluated a Chi-square $\left(\chi^{2}\right)$ for each progenitor model when compared to all available observations. Each observation-the maximum bolometric luminosity, bolometric luminosity measurements more than 60 days after maximum, and velocity measurements during the plateau phase-contributed equally to $\chi^{2}$ according to its error. Each model was then weighted by the probability of observing the calculated value of $\chi^{2}$, and binned in a histogram. The confidence regions drawn contain the stated fractions of the total probability of all models calculated. There may be additional constraints on the actual distribution of these parameters realized in nature, which we do not include in the sampling or weighting of different possible combinations of parameters. For example, SN Ia explosion physics should constrain the relative abundances of various elements in the ejecta; our constraints rely only on the kinematics of the explosion given the nuclear energy released. Due to this and other potential limitations, we therefore do not claim to pick out a single set of values which is most likely, nor to reproduce the detailed shapes of the distributions of underlying quantities. However, this approximate method should allow us to rule out untenable models. The size of the confidence regions, interpreted loosely, should reflect the uncertainty on the measurements themselves.

Figure 10 shows the first-pass results of this procedure for the case $\alpha=1.3$. The constraints are surprisingly tight: the data are consistent with a model containing $2.18 \pm 0.15 M_{\odot}$ of central ejected material, with $1.55 \pm 0.09 M_{\odot}$ of the mass being ${ }^{56} \mathrm{Ni}$, wrapped in a $\mathrm{C} / \mathrm{O}$ envelope of mass at least $0.3 M_{\odot}$. The shell of reverse-shocked SN ejecta contains about a third of the central ejected mass, or some $0.7-0.9 M_{\odot}$ of material. The minimum Chi-square value is $\chi_{v}^{2}=25.1 / 27=0.93$, indicating that the model is able to satisfy all available constraints. Chandrasekharmass progenitors, and progenitors with no envelopes, are ruled out at high significance. We place a lower limit of $2.05 M_{\odot}$ ( $99 \% \mathrm{CL}$ ) on the total mass of the system, and $0.1 M_{\odot}$ at similar confidence on the mass of the envelope.

The upper-right-hand plot in Figure 10 illustrate the tradeoff between unburnt, initially bound carbon/oxygen in the SN ejecta and more loosely bound carbon/oxygen in the surrounding envelope. An envelope turns out to be much more effective than bound unburnt material in producing the low velocities observed in the SN. The slope of the contour shows that about 4 times as much bound material is required to remain unburnt (decreasing the released nuclear energy for a given binding energy) as is needed to produce the same effect in an envelope (decelerating initially fast-moving ejecta in a collision). The inferred shell/envelope mass is therefore relatively insensitive to the amount of material left unburned in the $\mathrm{SN}$ explosion.

Given that $C_{\text {II }} \lambda 6580$ is the only visible carbon line in the velocity-plateau spectra around $B$-band maximum, and is detected only weakly, it seems unlikely that a great deal of unburned carbon/oxygen is mixed into the shell. Our observations can probably be adequately reproduced by setting $f_{\mathrm{CO}}=0$, so that all the unburned material is required to lie in the envelope (which must then have a mass of around $0.3 M_{\odot}$ ). This is more in line with the hydrodynamic behavior of the DET2ENVN models, and with our understanding of the physics of normal $\mathrm{SNe} \mathrm{Ia}$, in which almost all of the material in the original white 

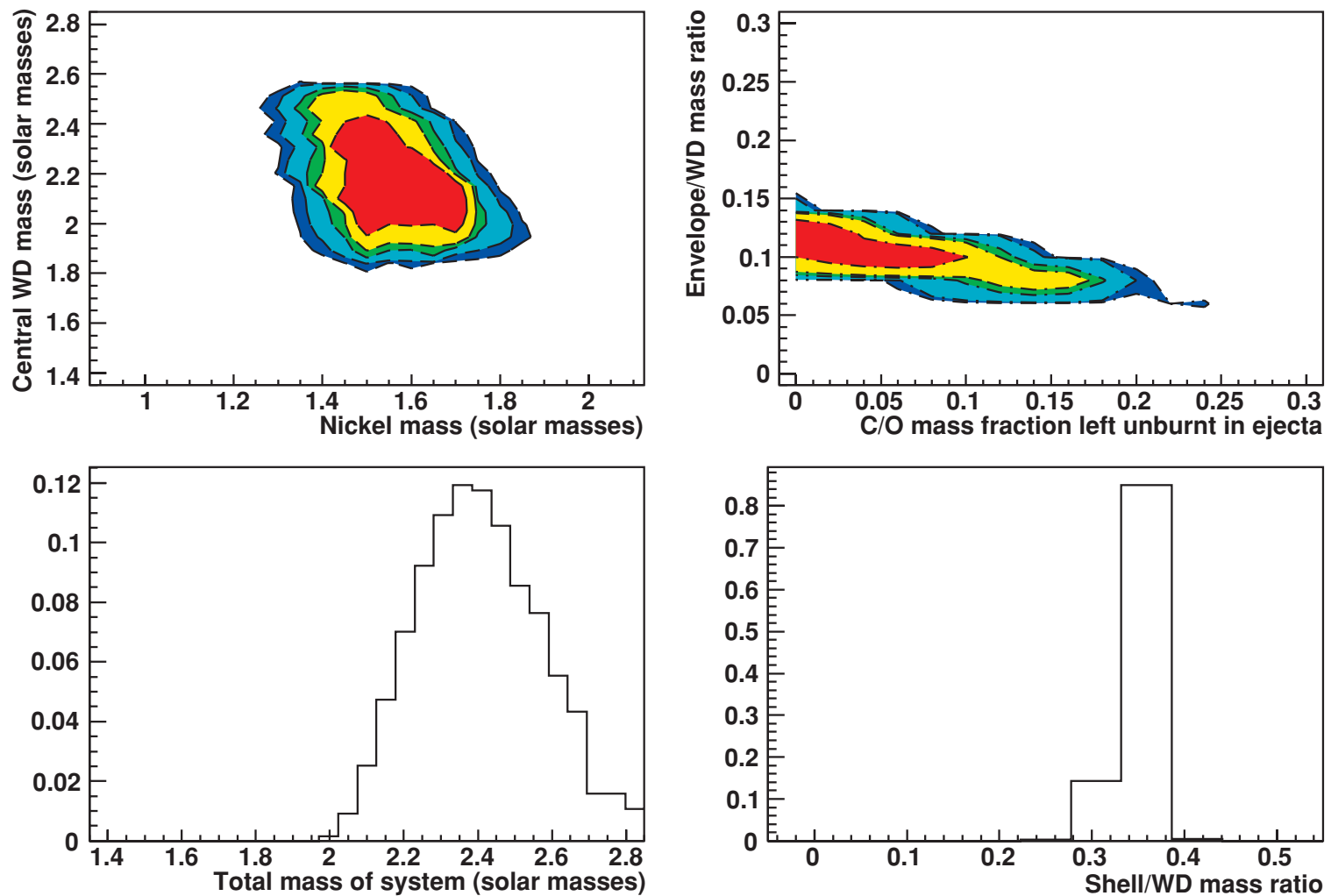

Figure 10. Constraints on progenitor models for SN 2007if, assuming $\alpha=1.3$ as for DET2ENVN and allowing for a floating fraction of unburned carbon/oxygen mixed into the shell of reverse-shock material. Colored regions are $68 \%$ (red), 90\%, 95\%, 99\%, and 99.7\% CL (blue). Top left: white dwarf mass $M_{\mathrm{WD}}$ vs. nickel mass $M_{56} \mathrm{Ni}$ in solar masses. Top right: envelope mass fraction $f_{\text {env }}$ vs. carbon-oxygen fraction $f_{\mathrm{CO}}$. Bottom left: probability distribution for the total mass $M_{\mathrm{tot}}=M_{\mathrm{WD}}+M_{\mathrm{env}}$. Bottom right: probability distribution for the fraction of ejected mass $f_{\mathrm{sh}}=M_{\mathrm{sh}} / M_{\mathrm{WD}}$ of material in the dense shell of reverse-shocked ejecta.

(A color version of this figure is available in the online journal.)

dwarf is burned in the explosion. Figure 11 shows how the mass constraints change when we set $f_{\mathrm{CO}}=0$; the low- ${ }^{56} \mathrm{Ni}$ models composed mainly of nickel and carbon/oxygen are excluded here. When the whole of $M_{\mathrm{wD}}$ must be burnt, much more additional mass must be included to increase the binding energy sufficiently to reproduce the appropriate value of $v_{e}$. Under these assumptions, the $99 \%$ CL lower limit on the system mass shifts upward to $2.20 M_{\odot}$.

The results of varying $\alpha$ are shown in Table 3. The allowed region of parameter space shows a tight anticorrelation between the central white dwarf mass and the ${ }^{56} \mathrm{Ni}$ mass. This intriguing behavior appears to result from the shape of the bolometric light curve: models with lower ${ }^{56} \mathrm{Ni}$ mass favor higher values of $t_{0}$, i.e., the gamma-ray escape fraction at a given time is lower in these models. It is interesting that the data seem to prefer $\alpha=1.3$ even with no outside input, providing further confirmation that this is a good choice for interpretation of the data.

\subsection{The Impact of Uncertain Reddening}

The analysis in the previous section was carried out assuming $E(B-V)_{\text {host }}=0$. If the $\mathrm{SN}$ is reddened by dust, we might expect the luminosity and the ${ }^{56} \mathrm{Ni}$ mass to be higher, and to produce a higher total mass. The other observations limit the extent of this effect, since an increased mass also leads to changes in the kinetic energy and density structure. Given the hard upper mass limit of $2 M_{\mathrm{Ch}}$ in our modeling, applying an uncertain reddening correction has little impact on our final mass constraints, but if certain reddening scenarios are shown to be incompatible with our observations, we can rule them out, even without recourse to the upper limit on Na I D absorption.
To constrain possible reddening scenarios, we repeated the analysis of Section 5.5 for the case $\alpha=1.3$, generating new bolometric light curves which had been reddened according to various prescriptions. The results are shown in Figure 12. These contours represent, in effect, different slices through the probability density in parameter space. We normalize the probability density displayed in each contour plot to the total probability in the zero-reddening case. This allows us to see how the relative total number of grid points which satisfy the observations changes as the reddening is varied. For example, the $68 \%$ contour in each of the plots is set at a level which encloses $68 \%$ of the probability in the zero-reddening case.

We find that increasing degrees of reddening shift the allowed contours to higher ${ }^{56} \mathrm{Ni}$ mass, as expected, although the total mass is not strongly affected. Our observations indicate $A_{V}<$ 0.38 (99\% CL), and are therefore incompatible with cases in which the Lira relation holds with $E(B-V)_{\text {host }}=0.18$ and either $R_{V}=2.1$ or $R_{V}=3.1$. High extinction tends to restrict solutions to a very small region of parameter space, in which the ejecta are composed almost entirely of ${ }^{56} \mathrm{Ni}$. Intermediate cases with $E(B-V)=0.09$ are consistent with the data and possibly also marginally consistent with the Lira relation at the extremes of systematic deviation within the set for which it was validated (Folatelli et al. 2010).

We conclude that, while some of the red color of SN 2007if may be put down to dust, it is likely that the Lira relation overestimates the extinction, although the slope of the color evolution of SN 2007if is similar to normal SNe Ia in the range of light curve phases where the Lira relation holds. If true, this has impact for the other observed super-Chandrasehkar-mass 

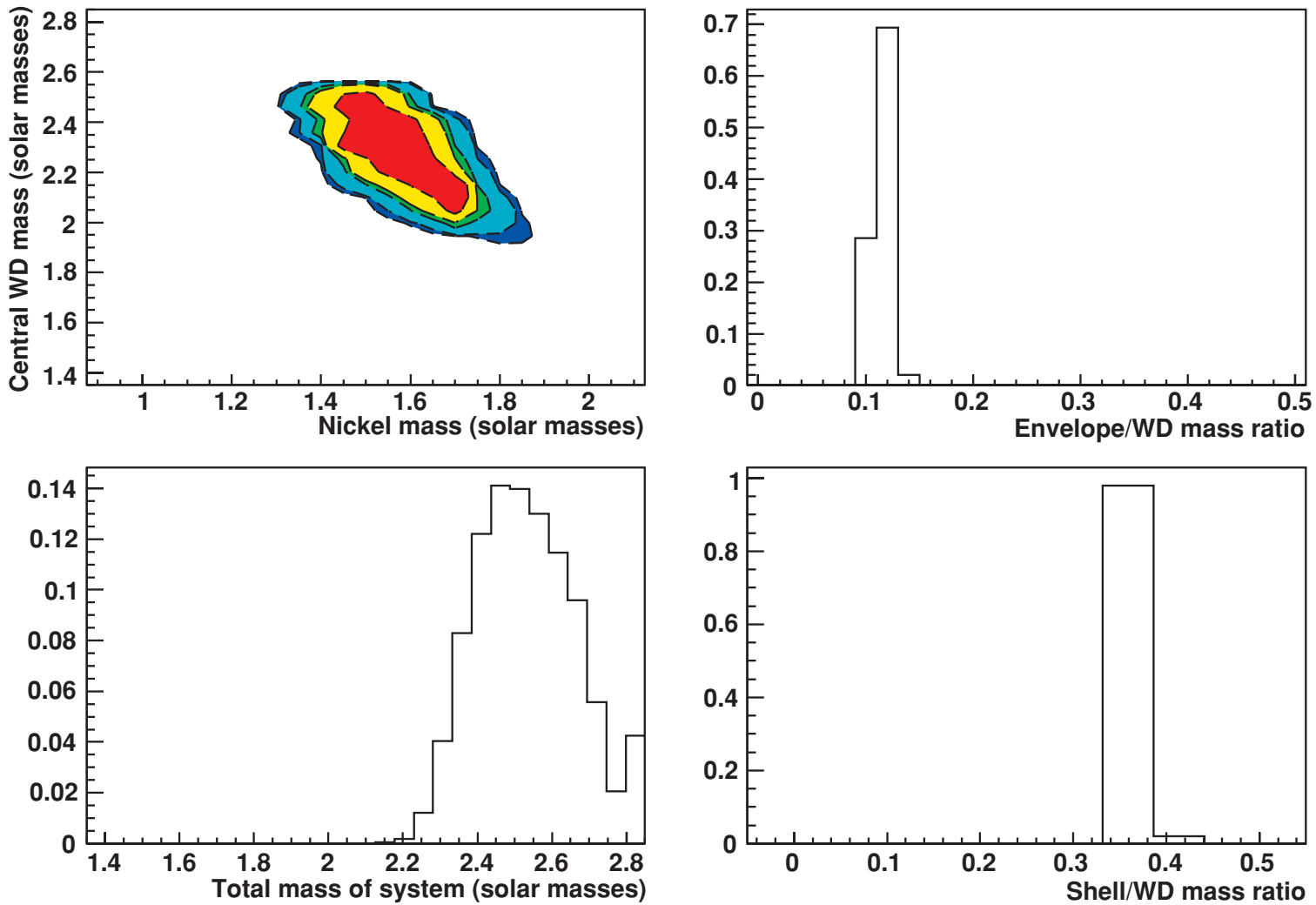

Figure 11. Constraints on progenitor models for SN 2007if, assuming $\alpha=1.3$ as for DET2ENVN and assuming that all carbon/oxygen must reside in the loosely bound envelope. Colored regions are 68\% (red), 90\%, 95\%, 99\%, and $99.7 \%$ CL (blue). Top left: white dwarf mass $M_{\mathrm{WD}}$ vs. nickel mass $M_{56} \mathrm{Ni}$ in solar masses. Top right: probability distribution for the envelope mass fraction $f_{\text {env }}$. Bottom left: probability distribution for the total mass $M_{\mathrm{tot}}=M_{\mathrm{WD}}+M_{\mathrm{env}}$. Bottom right: probability distribution for the fraction of ejected mass $f_{\mathrm{sh}}=M_{\mathrm{sh}} / M_{\mathrm{WD}}$ of material in the dense shell of reverse-shocked ejecta.

(A color version of this figure is available in the online journal.)

Table 3

Variation in Constraints with Model-dependent Parameters

\begin{tabular}{|c|c|c|c|c|c|c|c|c|c|}
\hline$\alpha$ & $E(B-V)_{\text {host }}$ & $R_{V \text {, host }}$ & $\rho_{c}\left(10^{9} \mathrm{~g} \mathrm{~cm}^{-3}\right)$ & $M_{\mathrm{tot}}\left(M_{\odot}\right)$ & $M_{\mathrm{WD}}\left(M_{\odot}\right)$ & $M_{\mathrm{Ni}}\left(M_{\odot}\right)$ & $f_{\text {env }}$ & $f_{\mathrm{sh}}$ & $\overline{\text { Prob. }^{a}}$ \\
\hline 0.80 & 0.00 & $\ldots$ & 3.00 & $\ldots$ & $\ldots$ & $\ldots$ & $\ldots$ & $\ldots$ & $<10^{-5}$ \\
\hline 0.90 & 0.00 & $\ldots$ & 3.00 & $2.26 \pm 0.06$ & $2.00 \pm 0.06$ & $1.84 \pm 0.06$ & $0.14 \pm 0.01$ & $0.39 \pm 0.02$ & 0.004 \\
\hline 1.00 & 0.00 & $\ldots$ & 3.00 & $2.28 \pm 0.09$ & $2.03 \pm 0.08$ & $1.78 \pm 0.07$ & $0.14 \pm 0.02$ & $0.38 \pm 0.02$ & 0.078 \\
\hline 1.20 & 0.00 & $\ldots$ & 3.00 & $2.36 \pm 0.15$ & $2.13 \pm 0.13$ & $1.63 \pm 0.09$ & $0.15 \pm 0.03$ & $0.36 \pm 0.03$ & 0.798 \\
\hline 1.30 & 0.00 & $\cdots$ & 3.00 & $2.41 \pm 0.17$ & $2.18 \pm 0.15$ & $1.55 \pm 0.09$ & $0.16 \pm 0.04$ & $0.34 \pm 0.03$ & 1.000 \\
\hline 1.40 & 0.00 & $\ldots$ & 3.00 & $2.45 \pm 0.18$ & $2.23 \pm 0.15$ & $1.48 \pm 0.08$ & $0.16 \pm 0.04$ & $0.32 \pm 0.03$ & 0.836 \\
\hline 1.30 & 0.09 & 2.1 & 3.00 & $2.42 \pm 0.11$ & $2.17 \pm 0.09$ & $1.87 \pm 0.09$ & $0.15 \pm 0.02$ & $0.36 \pm 0.03$ & 0.546 \\
\hline 1.30 & 0.09 & 3.1 & 3.00 & $2.45 \pm 0.09$ & $2.20 \pm 0.08$ & $1.98 \pm 0.10$ & $0.15 \pm 0.02$ & $0.36 \pm 0.03$ & 0.231 \\
\hline 1.30 & 0.18 & 2.1 & 3.00 & $2.50 \pm 0.06$ & $2.25 \pm 0.06$ & $2.03 \pm 0.10$ & $0.14 \pm 0.02$ & $0.36 \pm 0.03$ & 0.019 \\
\hline 1.30 & 0.18 & 3.1 & 3.00 & $\ldots$ & $\ldots$ & $\ldots$ & $\ldots$ & $\ldots$ & $<10^{-5}$ \\
\hline 1.30 & 0.00 & $\ldots$ & 0.03 & $2.72 \pm 0.07$ & $2.40 \pm 0.06$ & $1.61 \pm 0.08$ & $0.39 \pm 0.03$ & $0.40 \pm 0.03$ & 0.213 \\
\hline
\end{tabular}

Notes. Constraints on properties of models as a function of the (model-dependent) parameters $\alpha=L_{\mathrm{bol}} / L_{\mathrm{rad}}, E(B-V)_{\text {host }}, R_{V \text {,host }}$, and $\rho_{c}$. Columns, left to right: $\alpha$; assumed host extinction $E(B-V)$ and $R_{V}$; assumed central density $\rho_{c}$ of the white dwarf before explosion; mass of the white dwarf merger product $M_{\mathrm{WD}}$; ${ }^{56} \mathrm{Ni}$ mass synthesized in the explosion; ratio of envelope mass to white dwarf mass; ratio of shell mass to white dwarf mass; total probability, marginalized over all free parameters.

${ }^{\text {a }}$ Normalized to $\alpha=1.3$, no host extinction, $\rho_{c}=3 \times 10^{9} \mathrm{~g} \mathrm{~cm}^{-3}$ (fiducial analysis).

SN Ia candidates in which the Lira relation is used to correct for reddening, producing large uncertainties in the mass estimate from the SN luminosity alone for these SNe.

\subsection{The Impact of Uncertain Central Density}

One can also vary the central density $\rho_{c}$ of the SN progenitor at ignition, another input which we held fixed in our 

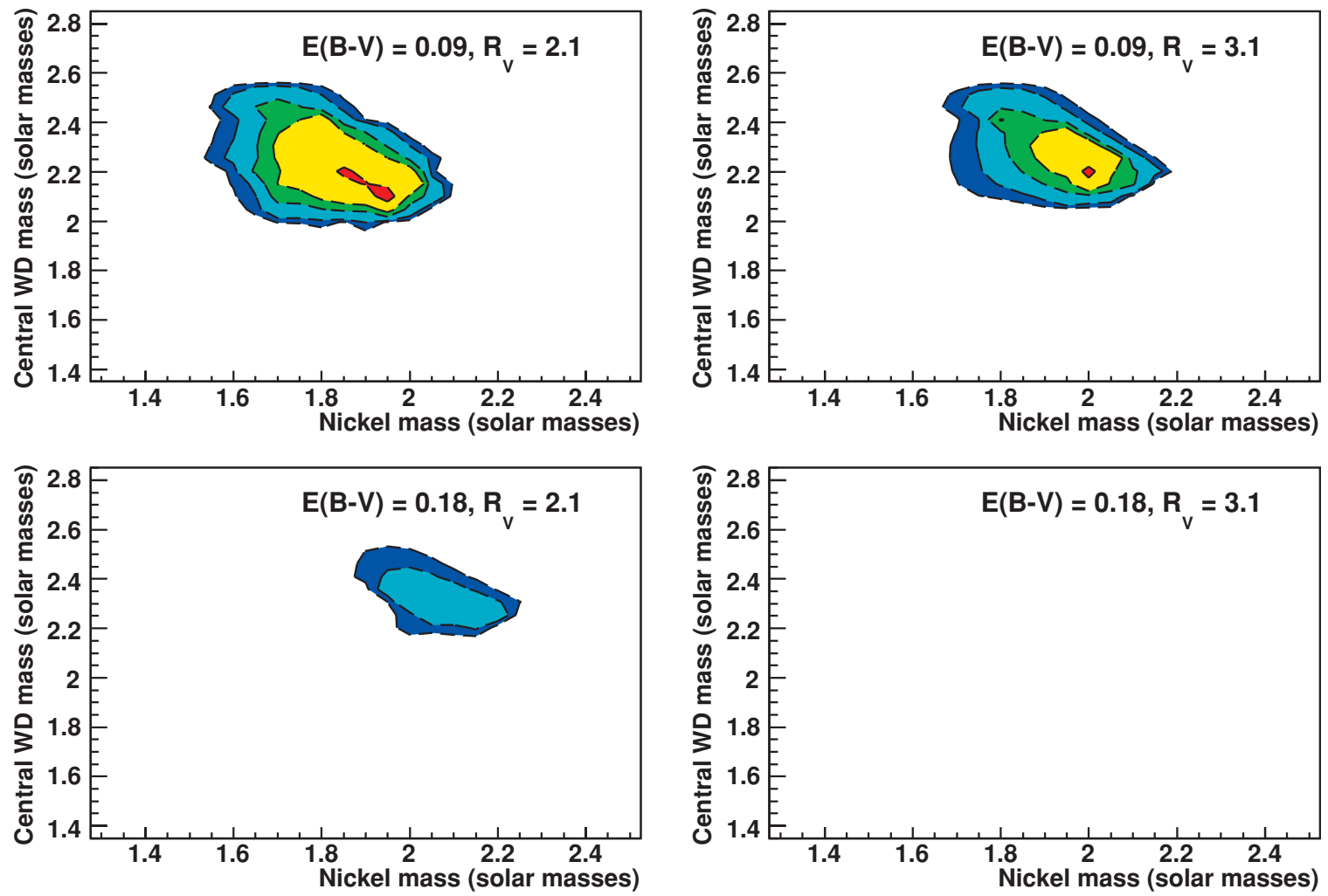

Figure 12. Constraints on progenitor model masses for SN 2007if assuming $\alpha=1.3$ (see e.g., upper left panel of Figure 10) for different possible reddening scenarios. Probability density is normalized to the zero-reddening case. Colored regions are $68 \%$ (red), 90\%, 95\%, 99\%, and 99.7\% CL (blue), Top left: $E(B-V)=0.09$, $R_{V}=2.1$. Top right: $E(B-V)=0.09, R_{V}=3.1$. Bottom left: $E(B-V)=0.18, R_{V}=2.1$. Bottom right: $E(B-V)=0.18, R_{V}=3.1$.

(A color version of this figure is available in the online journal.)

calculations above. The central density for carbon ignition in a Chandrasekhar-mass white dwarf is expected to be around $3 \times 10^{9} \mathrm{~g} \mathrm{~cm}^{-3}$ (Jeffery et al. 2006), and is our fiducial value in the above analysis. Maeda \& Iwamoto (2009) examine central densities as high as $10^{10} \mathrm{~g} \mathrm{~cm}^{-3}$, above which electron capture is expected to make collapse to a neutron star more likely than a SN Ia (Nomoto \& Kondo 1991; Yoon \& Langer 2005). The DET2ENVN models, which form the primary point of reference for our analysis in the context of a TD, actually have a much smaller central density, around $4 \times 10^{7} \mathrm{~g} \mathrm{~cm}^{-3}$.

There is therefore a dynamic range of at least two and a half orders of magnitude within which $\rho_{c}$ could be expected to vary. As with reddening, decreasing the central density can only increase the total reconstructed mass (by decreasing the specific binding energy), and so we expect it to have little impact on our final mass constraints. However, by seeing how our observations constrain allowed masses in different central density scenarios, we may be able to place a lower limit on the allowed density.

To this end, we repeat our analysis for $E(B-V)=0, \alpha=1.3$, sampling a range of central densities from $3 \times 10^{7} \mathrm{~g} \mathrm{~cm}^{-3}$ (close to that assumed for DET2ENVN) to our fiducial density. We normalize the probability densities to our fiducial model as in Section 5.6 above. The results are shown in Figure 13. We find that although very low values of the central density typical of DET2ENVN are disfavored, a wide range of possible values are compatible with the data. While the ${ }^{56} \mathrm{Ni}$ mass remains unaffected, the total mass increases, and the envelope mass fraction needed to decelerate the explosion to the observed velocities increases dramatically. At central densities typical of DET2ENVN, an envelope of nearly $1.0 M_{\odot}$ is needed to match the observations.
It is possible that other observables associated with our spectral time series (for example, the detailed chemical composition of the ejecta) could provide additional constraints on the central density. Such detailed modeling is beyond the scope of this paper, and awaits a refined theoretical description of super-Chandrasekhar-mass SN Ia progenitors, including hydrodynamic and radiative transfer simulations of the resulting explosions.

\section{ARE SHELLS COMMON IN SUPER-CHANDRASEKHAR-MASS EXPLOSIONS?}

Our detailed observations of SN 2007if have produced a relatively specific physical picture of the explosion, providing a framework within which to interpret SN 2003 fg-like SNe as a subclass. A summary of the observational properties of the various super-Chandrasekhar-mass SN Ia candidates found to date is given in Table 4; we discuss what is known about these SNe below, in the context of our findings. The data have been put on a uniform distance scale, and measurements and uncertainties for some quantities have been improved or corrected relative to the original sources employing the techniques we have applied to SN 2007 if.

Maeda \& Iwamoto (2009) argue that an aspherical explosion is needed to explain the high luminosity of SN 2003fg, based on the relatively fast decay time of its bolometric light curve $\left(t_{+1 / 2}=13\right.$ days $)$ and low peak velocity, and raise doubts about the high mass of the progenitor as interpreted in the bindingenergy framework of Howell et al. (2006). Conversely, they argue that the blue observed color, high velocities, and long bolometric decay time of SN $2006 \mathrm{gz}$ are consistent with a roughly spherical super-Chandrasekhar-mass progenitor. 

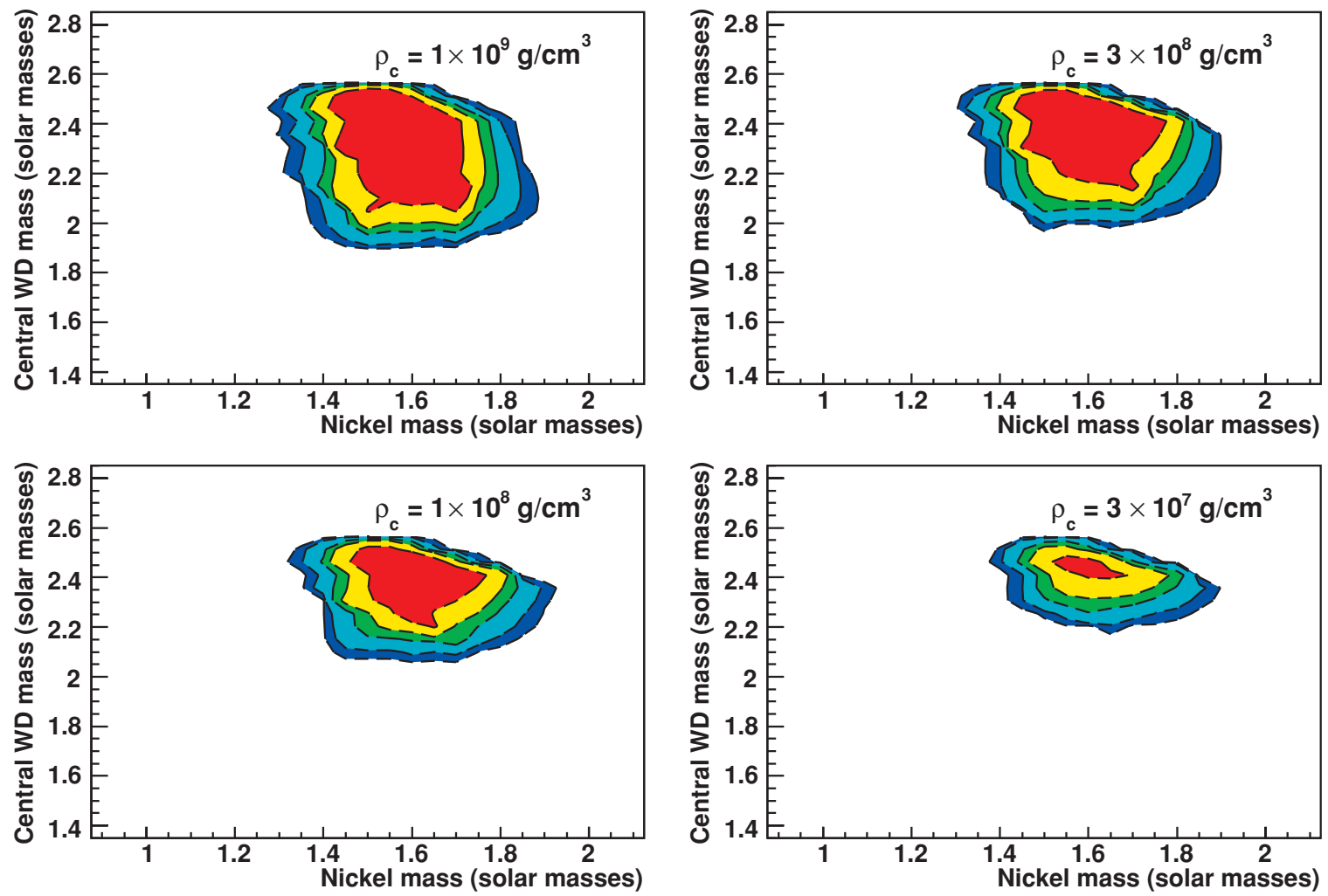

Figure 13. Constraints on progenitor model masses for SN 2007if, assuming $\alpha=1.3$ (see e.g., upper left panel of Figure 10) for different possible central densities. Probability density is normalized to that for the fiducial central density of $\rho_{c}=3 \times 10^{9} \mathrm{~g} \mathrm{~cm}^{-3}$. Colored regions are 68\% (red), 90\%, 95\%, 99\%, and 99.7\% CL (blue). Top left: $\rho_{c}=1 \times 10^{9} \mathrm{~g} \mathrm{~cm}^{-3}$. Top right: $\rho_{c}=3 \times 10^{8} \mathrm{~g} \mathrm{~cm}^{-3}$. Bottom left: $\rho_{c}=1 \times 10^{8} \mathrm{~g} \mathrm{~cm}^{-3}$. Bottom right: $\rho_{c}=3 \times 10^{7} \mathrm{~g} \mathrm{~cm}^{-3}$.

(A color version of this figure is available in the online journal.)

Table 4

Comparison of Super-Chandrasekhar-mass SN Ia Candidates

\begin{tabular}{|c|c|c|c|c|c|c|c|c|c|c|}
\hline \multirow{2}{*}{$\begin{array}{l}\text { Observational Property } \\
\mathrm{m}_{V} \\
\text { Galactic } \mathrm{E}(\mathrm{B}-\mathrm{V})\end{array}$} & \multicolumn{2}{|c|}{ SN 2003fg } & \multicolumn{2}{|c|}{ SN 2006gz } & \multicolumn{2}{|c|}{ SN 2007if } & \multicolumn{2}{|c|}{ SN 2009dc } & \multicolumn{2}{|c|}{$\begin{array}{c}\text { Population } \\
\text { Statistics }\end{array}$} \\
\hline & \multicolumn{2}{|c|}{0.013} & \multicolumn{2}{|c|}{0.063} & \multicolumn{2}{|c|}{0.079} & \multicolumn{2}{|c|}{0.071} & & \\
\hline $\mathrm{M}_{V}$ & \multicolumn{2}{|c|}{$-20.04 \pm 0.05^{\mathrm{a}}$} & \multicolumn{2}{|c|}{$-19.23 \pm 0.06^{b}$} & \multicolumn{2}{|c|}{$-20.39 \pm 0.04$} & \multicolumn{2}{|c|}{$-19.88 \pm 0.17^{\mathrm{c}}$} & & \\
\hline$B_{\max }-V_{\max }$ & \multicolumn{2}{|c|}{+0.14} & \multicolumn{2}{|c|}{$+0.03^{\mathrm{b}}$} & \multicolumn{2}{|c|}{+0.15} & \multicolumn{2}{|c|}{+0.21} & \multirow{2}{*}{\multicolumn{2}{|c|}{$9500(2100)$}} \\
\hline$v_{\mathrm{SiII}}\left(\mathrm{km} \mathrm{s}^{-1}\right)$ & \multicolumn{2}{|c|}{$\sim 8000$} & \multicolumn{2}{|c|}{$\sim 13000$} & \multicolumn{2}{|c|}{$\sim 9000$} & \multicolumn{2}{|c|}{$\sim 8000$} & & \\
\hline Host features & $\begin{array}{l}\text { emiss } \\
\text { in tide }\end{array}$ & $\begin{array}{l}\text { lines; } \\
\text { eature }\end{array}$ & $\begin{array}{r}\text { Scd spir } \\
\text { emissic }\end{array}$ & $\begin{array}{l}\text { no local } \\
\text { excess }^{\mathrm{d}}\end{array}$ & $\begin{array}{r}\text { emiss } \\
\mathrm{d}\end{array}$ & $\begin{array}{l}\text { lines; } \\
\text { arf }\end{array}$ & $\begin{array}{l}\text { S0; } \\
\text { form }\end{array}$ & $\begin{array}{l}\text { star } \\
\text { on? }\end{array}$ & & \\
\hline Host EW(Na I D) & $<1.7 \AA$ & $5 \% \mathrm{CL}$ & $0.33=$ & $0.03 \AA$ & $<0.12$ & $95 \% \mathrm{CL}$ & & & & \\
\hline$E(B-V)_{\text {Lira }}$ & 0.21 & 0.04 & 0.18 & 0.05 & 0.18 & 0.04 & 0.37 & 0.08 & & \\
\hline & & & Quantitic & Corrected & Host Extir & ion Using & I $D^{f}$ & & & \\
\hline & Shallow & Steep & Shallow & Steep & Shallow & Steep & Shallow & Steep & Shallow & Steep \\
\hline
\end{tabular}

Notes.

${ }^{a}$ Howell et al. (2006).

${ }^{\mathrm{b}}$ Hicken et al. (2007), with corrected distance modulus uncertainty.

c Yamanaka et al. (2009).

${ }^{\mathrm{d}}$ There is no $\mathrm{H} \alpha$ emission remaining in spectra of SN 2006gz after subtraction of an interpolated background.

e Inspection of SDSS images shows uniformly red color.

${ }^{\mathrm{f}}$ For SN 2003fg and SN 2007if the most probable value, EW(Na I D) = 0, was used.

Our above analysis of SN 2007if allows us to address the criticism of Maeda \& Iwamoto (2009) regarding the failures of a spherical geometry to describe the properties of SN 2003 fglike SNe. Their work assumed an essentially undisturbed ejecta 
structure close to an exponential in velocity, in which the peak Si II velocity was a suitable proxy for the kinetic energy of the explosion. We have seen that, for density structures with shells, this assumption is no longer true; powerful explosions with high kinetic energy can have low-velocity, slowly evolving photospheres.

A similar scenario may hold for SN 2003fg. Although it is difficult to test because of the limitations of the data, the existing observations of SN 2003 fg are broadly consistent with a SN 2007if-like scenario. The single published spectrum shows a low velocity which may or may not have been part of a plateau. SALT2 fits to various near-maximum subranges of the SNLS photometry result in values of $B-V$ at $B$-band maximum ranging between 0.03 and 0.14 , at odds with the published estimate $B-V=-0.15$ (Howell et al. 2006; Hicken et al. 2007). However, the published (statistical only) error bars on $B-V$ from Howell et al. (2006) probably underestimate the true error, given that there was no rest-frame $B$-band data at maximum light for SN 2003fg (D. A. Howell et al. 2009, private communication). A SALT2 fit to all available SNLS data gives $c=0.22 \pm 0.03$, or $B-V=+0.14$ at $B$-band maximum after correction for Galactic extinction; this color is consistent with the color we obtain from synthetic photometry of the published SN 2007if spectrum from SNLS (Howell et al. 2006). While the evidence is far from conclusive, the spectral match to SN 2007if near maximum light, and the arguments presented in Maeda \& Iwamoto (2009), suggest that SN 2003fg may be best interpreted as a super-Chandrasekhar-mass SN Ia with dense shell structure, like SN 2007if.

Likewise, SN 2009dc was also a very red SN with slow velocity evolution. Yamanaka et al. (2009) note that the very large value of the reddening, $E(B-V)_{\text {host }}=0.37$, derived from the Lira relation is at odds with the estimate $E(B-V)_{\text {host }}=0.15$ obtained from the shallow TBC relation. They adopt the latter value which, after correction, produces $B-V \sim+0.2$ for $\mathrm{SN}$ 2009 dc near peak. This is broadly similar to the intrinsic color we expect for SN 2007if. We note that Yamanaka et al. (2009) do not appear to correct for the efficiency factor $\alpha$ in their derivation of the ${ }^{56} \mathrm{Ni}$ mass of SN 2009dc; however, they also assume a fairly typical 20 day rise time for the $\mathrm{SN}$, when the true value (not strongly constrained by pre-maximum observations) is probably higher, given the SN's slow decline. These two errors have opposite and comparable effects on the derived ${ }^{56} \mathrm{Ni}$ mass, so it turns out that their estimate should in fact be accurate, and hence comparable to SN 2003fg. Taken together with the low, slowly evolving $\left(\sim 50 \mathrm{~km} \mathrm{~s}^{-1}\right.$ day $^{-1}$ to day +17$)$ Si II velocity, these observations are broadly compatible with a shell-structure density profile for the ejecta of SN $2009 \mathrm{dc}$. The strong detection of C II $\lambda 6580$ in the near-maximum-light spectra of SN 2009dc, at comparable velocity to Si II, suggests either that a larger fraction of carbon remained unburned than in SN 2007if, or that a pre-existing carbon/oxygen envelope may have been mixed into the ejecta after burning was complete.

The fact that the Lira relation appears to overcorrect the reddening for SN 2007if and SN 2009dc makes the interpretation of SN $2006 \mathrm{gz}$ problematic. The supposed high luminosity and mass of SN $2006 \mathrm{gz}$ rests on the Lira relation, corroborated by a low-significance detection of $\mathrm{Na}$ I and the assumption of a steep slope for the TBC relation, and the assumption $R_{V}=3.1$. The shallow TBC relation (adopted for SN 2009dc) gives $E(B-V)_{\text {host }}=0.05$, which would make SN 2006gz somewhat brighter and redder than average, but not unreasonably so for normal SNe Ia.
To further examine this question we use the measurements compiled in Table 4 to more closely examine the properties of this population under different extinction treatments. To aid in this comparison we have remeasured $\mathrm{EW}(\mathrm{Na}$ I D) from the host of SN 2006gz using the same approach as for SN 2007if, resulting in a significant $(5 \times)$ reduction in the uncertainty. By examining $M_{V}, B_{\max }-V_{\max }$, and the color excess, $E(B-V)_{\text {Lira }}$, with respect to the Lira relation we find that the properties of this population are most uniform when the shallow TBC relation is used to correct for host-galaxy extinction. The resulting rms in $M_{V}$ is only $0.40 \mathrm{mag}$, the $\mathrm{rms}$ in $B_{\max }-V_{\max }$ is only $0.08 \mathrm{mag}$, and the Lira color excess has a remarkably small rms of only 0.03 mag with this treatment. Use of the steep TBC relation doubles or triples the rms for each of these quantities, suggesting that it is disfavored by these data. Using the shallow TBC EW(Na I D) extinction correction places SN 2006gz at $M_{V}=-19.38$, well below the population mean of $\left\langle M_{V}\right\rangle=-20.02$. (In fact, without SN 2006gz the mean brightness of the population increases to $\left\langle M_{V}\right\rangle=-20.26$.)

Other indirect evidence also points to a lower mass and intrinsic luminosity for SN $2006 \mathrm{gz}$. Although the decay time of the SN 2006gz light curve is long, the rise time is short, lowering the ${ }^{56} \mathrm{Ni}$ mass estimate relative to what one might expect given the event's $\Delta m_{15}(B)$. Late-time spectroscopy and photometry of SN 2006gz (Maeda et al. 2009) do not provide strong evidence for a large mass of ${ }^{56} \mathrm{Ni}$ in the explosion. The Si II velocity at maximum is typical of normal SNe Ia, which suggests neither a high binding energy nor a very massive shell. While the plateau in the Si II velocity for SN $2006 \mathrm{gz}$ supports the existence of an envelope, as Hicken et al. (2007) suggest, the high velocity of the supposed plateau $\left(13,000 \mathrm{~km} \mathrm{~s}^{-1}\right)$ and its short duration, as well as the blue color of the SN relative to the SN 2003fg-like $\mathrm{SNe}$, argue for an envelope with mass only about $3 \%$ of the white dwarf mass $\left(0.04 M_{\odot}\right.$ for $\left.M_{\mathrm{WD}}=M_{\mathrm{Ch}}\right)$. Thus, while SN 2006gz might have been a DD merger and does show some evidence for a low-mass shell, it seems to be different from the red, unambiguously overluminous SN 2003fg-like SNe. If the short rise times of the PDD models of Höflich \& Khohklov (1996) relative to other explosion mechanisms are realized in nature, SN 2006gz may well have been a Chandrasekhar-mass PDD; the models PDD9 and PDD1a compare favorably.

In short, comparison of SN 2007if with other events suggests the following about SN 2003fg-like SNe (excluding SN 2006gz).

1. The TD scenario, in which the high-velocity ejecta are decelerated by an envelope, provides a cleaner explanation for the low photospheric velocities than a high binding energy alone, and it does so in the context of a nearly spherical explosion.

2. The presence of a shell also explains the long rise times and red colors of these events.

3. While the late-time color evolution of SN 2003 fg-like SNe appears to have a slope similar to the Lira relation for normal SNe Ia, the assumption that the intrinsic colors of these events at late times are similar to normal SNe Ia probably leads to an overestimate of the reddening.

More detailed analysis for these three $\mathrm{SNe}$ can be used to determine likely values for the progenitor masses, allowing for the possibility of shells, and (for TDs) to constrain the mass of the envelope. Such an exercise on a representative sample of $\mathrm{SNe}$ may be helpful in refining theoretical models of the DD merger process. 
Finally, we note that as an extremely faint system, the host of SN 2007if is most likely of low mass and low metallicity. The tidal tail in which SN $2003 \mathrm{fg}$ occurred may have similar properties. In contrast, SN $2006 \mathrm{gz}$ occurred in a luminous spiral and SN 2009dc occurred in an apparently quiescent elliptical galaxy. Thus, it remains unclear what role the progenitor metallicity may play in producing such events.

\section{CONCLUSIONS}

Our observations of SN 2007if provide detailed observations of the evolution of a candidate super-Chandrasekhar-mass SN Ia event, allowing new constraints on their progenitors. SN 2007if is the brightest $\mathrm{SN}$ Ia yet discovered, with an inferred ${ }^{56} \mathrm{Ni}$ mass of $1.6 \pm 0.1 M_{\odot}$; the near-IR light curves demonstrate evidence for a large fraction of iron-peak elements in the ejecta, some of which may have been mixed into the shell. The slow Si II velocity evolution near maximum is inconsistent with "normal" SN Ia photospheric evolution of expanding ejecta with a nearly exponential density structure, and is more readily interpreted as evidence for an overdense shell in the ejecta. Further evidence for an unusual density structure in the ejecta comes from the latetime bolometric light curve, which implies a higher gamma-ray escape fraction than one might expect for a progenitor with normal photospheric evolution. Interpreting the observations in the context of a TD model representative of a DD merger (as in the DET2ENVN models of Khokhlov et al. 1993), we use the SN 2007if bolometric light curve to establish the first constraint on the total mass for a super-Chandrasekhar-mass SN Ia progenitor. Our mass estimate should strictly be construed as a lower bound, since reddening by dust and a low central density will both result in a higher mass; however, extreme compositions and unrealistically high masses, at the edge of the allowed parameter space, result if either the reddening or the central density are very different from those used in our fiducial analysis in Section 5.5.

Better models of the progenitors and explosions of $\mathrm{SN}$ 2003 fg-like SNe Ia are urgently needed, mainly because of the theoretical limits on the existence of supermassive white dwarfs (Piro 2008; Chen \& Li 2009) which provide possible initial conditions for a SN 2003fg-like explosion. In our work, we nevertheless assume the existence of a central supermassive white dwarf and use the binding energy formula of Yoon \& Langer (2005). This formula has been validated only for $M<2.0 M_{\odot}$, but other works have performed similar extrapolations (e.g., Jeffery et al. 2006) simply because no appropriate alternative exists for modeling hypothetical explosions of supermassive white dwarfs.

Further uncertainty relates to the properties of the carbon/ oxygen envelope. The envelope considered in DET2ENVN and in this work is relatively small in extent $\left(R \sim 10^{10} \mathrm{~cm}\right)$, so the shock interaction which produces the shell can be approximated as occurring instantaneously, with the ejecta in homologous expansion thereafter. Our simple modeling suggests that a large fraction of the kinetic energy (up to $20 \%$ of the initial value, or a few $10^{50} \mathrm{erg}$ ) must be dissipated in the transition from explosion to the final state with fully developed shell. If the envelope is more diffuse, the shock interaction and conversion of kinetic energy would proceed over a longer period of time, with some of the energy released at optical wavelengths. The interaction could produce a "pseudo-continuum" similar to those seen in SNe IIa (Hamuy et al. 2003; Wood-Vasey et al. 2004; Aldering et al. 2006; Prieto et al. 2007), without the telltale $\mathrm{H}$ and $\mathrm{He}$ emission, but possibly including $\mathrm{C}$ and $\mathrm{O}$ emission, toplighting absorption from those elements in the SN atmosphere (Branch et al. 2000). This could explain the blue, relatively featureless continuum seen in SN 2007if at -9 days. It would also contribute to the long rise time and high luminosity of the event, thus inflating our derived ${ }^{56} \mathrm{Ni}$ mass. Although we find this scenario unlikely, very early-phase observations of future events similar to SN 2007if, along with theoretical calculations of synthetic spectra of such an interaction, will be needed in order to constrain it. However, even if our ${ }^{56} \mathrm{Ni}$ mass is too high, the uniformity of the optical spectra at late times provide some evidence that the interaction produces negligible late-time luminosity. The lower limit on the total ejected mass from the late-time bolometric light curve should therefore remain secure, since the anticorrelation shown in Table 3 and Figure 11 requires higher total masses for models with lower ${ }^{56} \mathrm{Ni}$ mass.

More definitive statements about the progenitor mass and observational properties await detailed radiative transfer calculations. These will require more accurate density structures of super-Chandrasekhar-mass progenitors, starting from appropriate explosion models-which may in turn require initial conditions appropriately chosen, e.g., from simulations of the dynamics of DD inspiral events. The theoretical uncertainty on the parameter $\alpha$ relating the radioactivity and bolometric luminosities at maximum light presented another limiting factor, in light of the degeneracy between the ${ }^{56} \mathrm{Ni}$ mass and the total mass for a particular bolometric light curve. Clearer predictions for the rise time and intrinsic color of super-Chandrasekhar-mass $\mathrm{SNe}$ Ia, with and without envelopes or shell structure, would also be helpful in understanding these events.

A larger sample of SN 2003fg-like SNe is also needed to assess whether velocity plateaus truly are generic in these objects; this will require fairly intensive spectroscopic followup as quickly after explosion as possible. Our extensive spectral time series, including one of the earliest (the earliest if SN $2006 \mathrm{gz}$ is not included in this subclass) known spectrum of a SN 2003fg-like SN Ia (-9 days), should aid in the prompt identification of these events for future follow-up, especially in cases where redshifts of faint host galaxies are difficult to obtain. Optical/NIR light curves out to 100 days past maximum light would be useful to confirm the high mass in these explosions. NIR observations could be particularly useful for reducing the uncertainty in the dust extinction (and thereby testing the Lira relation) for SN 2003 fg-like SNe, and for determining the distribution of iron-peak elements for these objects. Modeling of the photospheric (Mazzali et al. 2007, 2008; Gal-Yam et al. 2009) and nebular (e.g., Mazzali et al. 1997; Maeda et al. 2009) spectral time series would be useful to provide independent constraints on the ${ }^{56} \mathrm{Ni}$ mass, the total ejected mass, and the specific products of nuclear burning in super-Chandrasekharmass $\mathrm{SNe}$, allowing us to learn more about the details of the explosion.

These detailed results will allow us to begin to connect SN 2003fg-like SNe to potential counterparts among "normal" $\mathrm{SNe}$ Ia. Construction of late-time bolometric light curves for a representative sample of less luminous velocity-plateau and/or LVG (Benetti et al. 2005) SNe Ia could enable new measurements of the ejected masses and shell mass fractions for these objects, presenting strong new constraints on their progenitors. Comparison of these SNe with SN 2003fg-like $\mathrm{SNe}$ could also enable observational methods to isolate or "tag" a subclass demanding different treatment when measuring luminosity distances. Quimby et al. (2007) caution that shellstructure $\mathrm{SNe}$ should be treated differently from normal $\mathrm{SNe}$ 
Ia in cosmology fits, having intrinsically redder colors and violating the typical width-luminosity relations. Howell et al. (2006) give a similar warning relating to a possible class of super-Chandrasekhar-mass progenitors with smaller inferred masses than SN 2003fg. Limiting the impact of such scenarios is crucial for the success of future (Stage III \& IV) dark energy experiments. Fortunately, with the addition of SN 2007if, the properties of this subclass are becoming more clear, and their clear spectral peculiarity establishes a means to recognize such events.

The authors are grateful to the technical and scientific staffs of the University of Hawaii $2.2 \mathrm{~m}$ telescope, the W. M. Keck Observatory, and Palomar Observatory, to the QUEST-II collaboration, and to HPWREN for their assistance in obtaining these data. Some of the data presented herein were obtained at the W. M. Keck Observatory, which is operated as a scientific partnership among the California Institute of Technology, the University of California, and the National Aeronautics and Space Administration. The Observatory was made possible by the generous financial support of the W. M. Keck Foundation. The authors wish to recognize and acknowledge the very significant cultural role and reverence that the summit of Mauna Kea has always had within the indigenous Hawaiian community. We are most fortunate to have the opportunity to conduct observations from this mountain. This work was supported by the Director, Office of Science, Office of High Energy Physics, of the U.S. Department of Energy under Contract No. DE-AC02-05CH11231; by a grant from the Gordon \& Betty Moore Foundation; and in France by support from CNRS/IN2P3, CNRS/INSU, and PNC. R.S. acknowledges support from National Science foundation grant 0407297. Y.C. acknowledges support from a Henri Chretien International Research Grant administrated by the American Astronomical Society, and from the France-Berkeley Fund. A.G.-Y. is supported by the Israeli Science Foundation, an EU Seventh Framework Programme Marie Curie IRG fellowship and the Benoziyo Center for Astrophysics, Minerva program, a research grant from the Peter and Patricia Gruber Awards, and the William Z. and Eda Bess Novick New Scientists Fund at the Weizmann Institute. This research used resources of the National Energy Research Scientific Computing Center, which is supported by the Director, Office of Science, Office of Advanced Scientific Computing Research, of the U.S. Department of Energy under Contract No. DE-AC02-05CH11231. We thank them for generous allocation of storage and computing time. HPWREN is funded by National Science Foundation Grant Number ANI-0087344, and the University of California, San Diego. IRAF is distributed by the National Optical Astronomy Observatories, which are operated by the Association of Universities for Research in Astronomy, Inc., under cooperative agreement with the National Science Foundation. The spectra of SN 1999ee were obtained through the SUSPECT Supernova Spectrum Archive, an online database maintained at the University of Oklahoma, Norman. We thank Andy Howell for providing photometry and the near-maximum spectrum of SN 2003fg, and Masayuki Yamanaka for providing Si II and C II velocity measurements derived from SN 2009dc spectra. The SMARTS $1.3 \mathrm{~m}$ observing queue receives support from NSF grant AST-0707627. We thank John Holtzman and Jon Cough for their assistance in photometrically monitoring our SN candidates with the NMSU $1 \mathrm{~m}$ telescope. We thank Dan Birchall for his assistance in collecting data with SNIFS, and for his helpful commentary on, and proofreading of, the manuscript. We also thank Charles Bailyn, Richard Pogge, and Kevin Krisciunas for their assistance in characterizing the ANDICAM system throughput, and Daniel Kasen and Alan Calder for helpful discussions.

Facilities: UH:2.2m, Hale(PO:5.0m), PO:1.2m, CTIO:1.5m

\section{REFERENCES}

Akerlof, C., et al. 2007, CBET, 1059, 2

Aldering, G., Knop, R., \& Nugent, P. 2000, AJ, 119, 2110

Aldering, G., et al. 2002, Proc. SPIE, 4836, 61

Aldering, G., et al. 2006, ApJ, 650, 510

Arnett, W. D. 1982, ApJ, 253, 785

Bacon, R., Emsellem, E., Copin, Y., \& Monnet, G. 2000, in ASP Conf. Ser. 195, Imaging the Universe in Three Dimensions, ed. W. van Breugel \& J. Bland-Hawthorn (San Francisco, CA: ASP), 173

Bacon, R., et al. 1995, A\&AS, 113, 347

Bacon, R., et al. 2001, MNRAS, 326, 23

Bailey, S., et al. 2009, A\&A, 500, L17

Baltay, C., et al. 2007, PASP, 119, 1278

Benetti, S., et al. 2005, ApJ, 623, 1011

Bertin, E., \& Arnouts, S. 1996, A\&AS, 117, 393

Bertin, E., et al. 2002, in ASP Conf. Ser. 281, Astronomical Data Analysis Software and Systems XI, ed. D. A. Bohlender, D. Durand, \& T. H. Handley (San Francisco, CA: ASP), 228

Bessell, M. S. 1990, PASP, 102, 1181

Branch, D., Baron, E. A., \& Jeffery, D. A. 2003, in LNP 598, Supernovae and Gamma-Ray Bursters, ed. K. Weiler (Berlin: Springer), 47

Branch, D., Jeffery, D. J., Blaylock, M., \& Hatano, K. 2000, PASP, 112, 217

Branch, D., et al. 2008, PASP, 120, 135

Buton, C. 2009, Ph.D. thesis, Université de Lyon

Cardelli, J. A., Clayton, G. C., \& Mathis, J. S. 1988, ApJ, 329, L33

Chen, W. C., \& Li, X. D. 2009, ApJ, 702, 686

Conley, A., et al. 2006, AJ, 132, 1707

Dobbie, P. D., et al. 2006, MNRAS, 373, L45

Dobbie, P. D., et al. 2009, MNRAS, 395, 2248

Folatelli, G., et al. 2010, AJ, 139, 120

Frogel, J. 1998, PASP, 110, 200

Gal-Yam, A., et al. 2009, Nature, 462, 624

Guy, J., et al. 2007, A\&A, 466, 11

Hamuy, M., et al. 2002, AJ, 124, 417

Hamuy, M., et al. 2003, Nature, 424, 651

Hicken, M., et al. 2007, ApJ, 669, L17

Hillebrandt, W., Sim, S. A., \& Röpke, F. K. 2007, A\&A, 465, L17

Höflich, P., \& Khohklov, A. 1996, ApJ, 457, 500

Howell, D. A., et al. 2006, Nature, 443, 308

Howell, D. A., et al. 2009, ApJ, 691, 661

Iben, I., \& Tutukov, A. V. 1984, ApJS, 54, 335

Jeffery, D. J. 1999, arXiv:astro-ph/9907015

Jeffery, D. J., \& Branch, D. 1990, in Supernovae, Jerusalem Winter School for

Theoretical Physics, ed. J. C. Wheeler, T. Piran, \& S. Weinberg (Singapore: World Scientific), 149

Jeffery, D. J., Branch, D., \& Baron, E. 2006, arXiv:astro-ph/0609804

Jha, S., et al. 2007, ApJ, 659, 122

Kasen, D. 2006, ApJ, 649, 939

Kasen, D., Nugent, P., Thomas, R. C., \& Wang, L. 2004, ApJ, 610, 876

Kasen, D., \& Woosley, S. E. 2007, ApJ, 656, 661

Khokhlov, A., Müller, E., \& Höflich, P. 1993, A\&A, 270, 223

Kim, A. G., Linder, E. V., Miquel, R., \& Mostek, N. 2004, MNRAS, 347, 909

Landolt, A. U. 1992, AJ, 104, 340

Lantz, B., et al. 2004, Proc. SPIE, 5249, 146

Linder, E. V. 2006, Phys. Rev. D, 74, 103518

Maeda, K., \& Iwamoto, K. 2009, MNRAS, 394, 239

Maeda, K., et al. 2009, ApJ, 690, 1745

Mazzali, P. A., Danziger, I. J., \& Turatto, M. 1995, A\&A, 297, 509

Mazzali, P. A., et al. 1997, MNRAS, 284, 151

Mazzali, P. A., et al. 2001, ApJ, 547, 988

Mazzali, P. A., et al. 2007, Science, 315, 825

Mazzali, P. A., et al. 2008, MNRAS, 386, 1897

Mink, D. 2006, in ASP Conf. Ser. 351, Astronomical Data Analysis Software and Systems XV, ed. C. Gabriel, C. Arviset, D. Ponz, \& E. Solano (San Francisco, CA: ASP), 204

Monet, D. G., et al. 2003, AJ, 125, 984

Nadyozhin, D. K. 1994, ApJS, 92, 527

Nomoto, K., \& Kondo, Y. 1991, ApJ, 367, L19

Nugent, P. E., Kim, A. G., \& Perlmutter, S. 2002, PASP, 114, 803 
Oke, J. B., et al. 1995, PASP, 107, 375

Perlmutter, S., et al. 1999, ApJ, 517, 565

Phillips, M. M., et al. 1999, AJ, 118, 1766

Piro, A. 2008, ApJ, 679, 616

Prieto, J., \& Depoy, D. 2006, CBET, 651, 1

Prieto, J. L., et al. 2007, AJ, submitted (arXiv:0706.4088)

Quimby, R., Höflich, P., \& Wheeler, J. C. 2007, ApJ, 666, 1083

Riess, A. G., et al. 1998, AJ, 116, 1009

Riess, A. G., et al. 1999, AJ, 118, 2675 (R99)

Schlegel, D. J., Finkbeiner, D. P., \& Davis, M. 1998, ApJ, 500, 525

Silverman, J. M., et al. 2010, MNRAS, submitted (arXiv:1003.2417)

Stritzinger, M., Leibundgut, B., Walch, S., \& Contardo, G. 2006, A\&A, 450, 241

Stritzinger, M., Suntzeff, N. B., Hamuy, M., Challis, P., Demarco, R., Germany, L., \& Soderberg, A. M. 2005, PASP, 117, 810
Sullivan, M., et al. 2006, AJ, 131, 960

Tanaka, M., et al. 2009, arXiv:0908.2057

Thomas, R. C., et al. 2007, ApJ, 654, L53

Thomas, R. C., et al. 2009, BAAS, 41, 464

Tody, D. 1993, in ASP Conf. Ser. 52, Astronomical Data Analysis Software and Systems II, ed. R. J. Hanisch, R. J. V. Brissenden, \& J. Barnes (San Francisco, CA: ASP), 173

Turatto, M., Benetti, S., \& Cappellaro, E. 2002, arXiv:astro-ph/0211219

van Dokkum, P. 2001, PASP, 113, 1420

Wang, X., et al. 2009, ApJ, 699, L139

Whelan, J., \& Iben, I. J. 1973, ApJ, 186, 1007

Wood-Vasey, W. M., et al. 2004, ApJ, 616, 339

Wright, E. L. 2006, PASP, 118, 1711

Yamanaka, M., et al. 2009, ApJ, 707, L118

Yoon, S.-C., \& Langer, N. 2005, A\&A, 435, 967 